\title{
Roles of solid effective stress and fluid-particle interaction force in modeling shear-induced particle migration in non-Brownian suspensions
}

\author{
Rashid Jamshidi $\odot$ \\ Department of Chemical Engineering, University College London, London WC1E 7JE, United Kingdom \\ Jurriaan J. J. Gillissen \\ Department of Mathematics, University College London, London WC1E 6BT, United Kingdom \\ Panagiota Angeli and Luca Mazzei $\oplus^{*}$ \\ Department of Chemical Engineering, University College London, London WC1E 7JE, United Kingdom
}

(Received 15 June 2020; accepted 16 December 2020; published 7 January 2021)

\begin{abstract}
The mixture, or suspension balance, model is the most frequently used to describe shear-induced particle migration in Newtonian fluids. The model suggests that neutrally buoyant particles migrate only if the effective stress tensor of the solid phase is nonuniform. In moderately dense suspensions, where direct particle contacts and interparticle forces are negligible, this tensor originates from the velocity fluctuations of the particles. Using Buyevich's constitutive equation, we show that the time required by these fluctuations to induce significant particle migration exceeds considerably the timescale of the process, obtained experimentally. In particular, the ratio between these two timescales is proportional to the reciprocal of the Reynolds number; hence, for vanishingly small values of the Reynolds number (the case on which this work focuses), particle velocity fluctuations cannot be responsible for particle migration. We conclude that, if direct particle contacts and interparticle forces are absent, particle migration must have another driver. In the literature, it has been suggested that this is the lubrication forces between the particles. On this assumption, Morris and Boulay advanced a closure for the solid effective stress tensor. This closure seems to predict well the migration process, but as discussed by P. R. Nott, E. Guazzelli, and O. Pouliquen, The suspension balance model revisited, Phys. Fluids 23, 043304 (2011), it presents some conceptual issues. We investigate this matter and show that lubrication forces can induce particle migration, and the tensor quantifying their effect can be closed by Morris and Boulay's equation; however, this tensor is not part of the effective stress tensor of the solid phase.
\end{abstract}

DOI: 10.1103/PhysRevFluids.6.014301

\section{INTRODUCTION}

Since the late 1970s, researchers have been investigating the migration of particles in Newtonian liquids, experimentally, theoretically, and computationally. This phenomenon is important in many industrial applications and laboratory measurements. For instance, in pharmaceutical and food manufacturing lines, where suspensions have to be kept as uniform as possible, migration is detrimental; similarly, in mining and oil and gas industries, where pipelines are long, it can cause severe problems, such as particle segregation and build-up. Migration also affects laboratory measurements,

*1.mazzei@ucl.ac.uk 
since in them mixtures must be uniform [1]. One familiar example is the measurement of the effective viscosity of a suspension. Indeed, it was during such a measurement that particle migration toward regions of low shear rate was first detected [2], a phenomenon known as shear-induced particle migration.

Shear-induced particle migration is a collective effect of the fluid-mediated interactions of several particles and occurs in (moderately) dense suspensions [3]. This process has nothing in common with the "tubular pinch" effect reported by Segré and Silberberg [4], which results from the interaction between an individual particle and the ambient fluid and originates from the inertia of the fluid in its motion relative to the particles. Shear-induced particle migration is not driven by inertia and also happens in mixtures with vanishingly small particle Reynolds number, even if the particles are not Brownian. In fact, these mixtures-where inertial and Brownian effects are negligible-are the target of our paper.

To describe the behavior of suspensions, and specifically shear-induced particle migration, most researchers have adopted the mixture model (e.g., Refs. [5-12]). This treats the suspension as an effective fluid and holds when the velocity fields of the fluid and solid phases relax to local equilibrium rapidly; more specifically, the time the fields require to equilibrate must be much shorter than the characteristic timescale of the flow $[13,14]$. This condition is usually met for suspensions of micron-size particles in very viscous fluids, the focus of our work. The mixture model consists of (i) a continuity equation and a linear momentum balance equation for the mixture, which yield its density and velocity fields, (ii) a continuity equation for the solid phase, which yields its volume fraction field, and (iii) a dynamical equation, which yields the migration velocity of the particles. This last equation features the effective stress tensor of the solid phase (e.g., Refs. [14,15]) and predicts that neutrally buoyant particles (the focus of this and most studies in this field) may migrate only if this tensor is nonuniform. So to predict the migration velocity, researchers need an accurate constitutive equation for this quantity.

Deriving such an equation is extremely challenging. The kinetic theory approach could in principle overcome the closure problem. Based on a generalization of the Boltzmann equation [16], this regards the solid effective stress tensor as a function of a granular temperature, a field related to the particle velocity fluctuations and governed by a pseudointernal energy balance equation that would complement the four balance equations of the mixture model previously discussed $[13,17,18]$. This method poses two significant challenges. The first is capturing in the pseudointernal energy balance equation all the relevant mechanisms generating and depleting particle velocity fluctuations or, equivalently, granular internal energy. The second is relating the granular temperature and solid effective stress tensor fields. These challenges limit the viability of this approach $[3,13,14,19]$.

Confronted with the difficulties that a fundamental approach poses, some research groups tackled the problem heuristically [20-22], developing the diffusive-flux model. This replaces the dynamical equation of the mixture model yielding the migration velocity of the particles with a closure for the migration velocity, derived via relatively simple arguments. This model gives good predictions in flows where the velocity and shear rate change in the same direction, its results matching closely various experimental data. Examples are pressure-driven laminar pipe flows and Couette flows. But when velocity and shear rate change in different directions, the model fails. For instance, in parallelplate rheometers the model predicts an inward (from high to low shear rates) particle migration, but experiments indicate that either no migration [23,24] or outward migration $[7,25,26]$ occurs; conversely, in cone-and-plate rheometers, where the shear rate field is uniform, the model predicts no migration, but experiments indicate that outward migration occurs [27]. Therefore, even if the diffusive-flux model is convenient, these drawbacks limit its applicability severely.

A third approach, which is neither entirely theoretical nor entirely heuristic, was proposed by Morris and Boulay [15]. To obtain the migration velocity of the particles, they did employ the dynamical equation of the mixture model featuring the solid effective stress tensor, but to close this tensor, they relied on heuristic arguments based on experimental evidence. So this approach does not involve a granular temperature field and does not require a pseudointernal energy balance equation. Morris and Boulay focused on curvilinear flows, showing that the presence of anisotropic normal 
stresses induced by the presence of the particles is sufficient to explain particle migration. If the parameters of their constitutive equation are properly tuned, then the closure predicts well steady-state solid concentration profiles in several viscometric flows and transient solid concentration profiles in wide-gap Couette flows. Its main limitation is that it holds only for viscometric flows. Furthermore, while this closure undoubtedly yields good results, its derivation poses a conceptual problem. Recently discussed by Nott et al. [28], this arises because Morris and Boulay ascribe to the solid effective stress tensor a contribution (the particle-presence stress tensor, which we will define and discuss in Sec. II and Appendix A) that belongs to the effective stress tensor of the fluid phase. The same problem, as Nott et al. [28] point out, is present in several works in this field-including the pioneering article of Nott and Brady [17], to which Morris and Boulay refer when deriving their constitutive equation. In light of this, one may rightly wonder how it is possible that the equation of Morris and Boulay predicts so well particle migration. This is a question we shall answer in this article.

Another heuristic closure of the solid effective stress tensor was suggested by Buyevich [3], who argued that the shear-induced fluctuations of the particle velocities are responsible for the migration of particles. The closure does not present the problem discussed above, has general validity, leads to an anisotropic solid effective stress tensor, and predicts well the solid volume fraction profile in a few fully developed viscometric flows [3,29]. However, in contrast to the closure of Morris and Boulay [15], it has been tested far less and has never been employed in transient conditions. Here we will test it in these conditions.

The idea that anisotropy in the solid phase normal stresses drives shear-induced particle migration is popular, but it has not been proven. This idea rests on experimental evidence, but rheological measurements inform on the effective stress tensor of the suspension-not of the solid phase. This important point has also been discussed by Nott et al. [28], who pointed out that the anisotropy in the normal stresses of the suspension has been wrongly interpreted in the literature as that of the normal stresses of the solid phase. More generally, many works incorrectly split, between the effective stress tensors of the fluid and solid phases, the several contributions making up the suspension effective stress tensor (e.g., Refs. [8,12,17,30-33]). This aspect has been reviewed by Jamshidi et al. [14], who based their analysis on the papers of Jackson [34] and Zhang and Prosperetti [35], landmark investigations that stand out for their rigor, clarity, and insight. In light of this, Nott et al. [28] disputed the notion that only normal stress differences in the solid effective stress tensor can induce particle migration, proving that (a part of) the mean force of interaction between the fluid and the particles can equally induce migration. In (moderately) dense suspensions, this term is related to the mean fluid-particle interaction force associated with the lubrication films between neighboring particles. Pursuing this idea, in this article we derive an expression for this force, showing that it relates to the effective stress tensor of the fluid phase, more specifically to the particle-presence stress associated with lubrication films. The resulting form of the particle migration velocity is consistent with that obtained from the constitutive equation of Morris and Boulay [15], an outcome that explains why their equation predicts well the migration process and that allows us to reinterpret their closure.

The paper is organized as follows. In Sec. II, we present the two-fluid and mixture models, focusing on the effective stress tensors of the fluid and solid phases and the terms constituting them. In Sec. III, we examine the constitutive equations for the solid effective stress tensor of Buyevich [3] and Morris and Boulay [15], highlighting their limitations and/or inconsistencies. In Sec. IV, following the idea suggested by Nott et al. [28], we illustrate that particle migration can be induced by the mean fluid-particle interaction force related to lubrication films and discuss how this result bears on the constitutive equation of Morris and Boulay [15]. The paper also includes three Appendixes. In the first, we briefly outline the derivation of the two-fluid equations of motion, offering more information about the effective stress tensors of the two phases and in particular about the particle-presence stress tensor. In the second, we connect our work to that of Batchelor [36], a landmark paper on the effective stress of suspensions, discussing how his particle stress tensor is related to the particle-presence stress tensor previously mentioned. In the last Appendix, we discuss the analysis of the steady wide-gap Couette flow presented in Morris and Boulay [15]. 


\section{THEORY}

This section presents the two-fluid and mixture models and discusses the expressions of their unclosed terms. In Appendix A, we briefly derive the averaged equations of motion of the two-fluid model, outlining the main passages and discussing some aspects that are closely related to our work. For more details, we refer to the literature [34,35,37].

\section{A. The two-fluid model}

Consider a suspension of identical particles with radius $a$ and density $\rho_{s}$ dispersed in an incompressible, isothermal, Newtonian liquid with density $\rho_{e}$ and constant viscosity $\mu_{e}$. For the fluid, the mass and linear momentum balance equations read:

$$
\begin{gathered}
\partial_{t} \varepsilon_{e}=-\partial_{\boldsymbol{x}} \cdot\left(\varepsilon_{e}\langle\boldsymbol{u}\rangle_{e}\right), \\
\rho_{e} \partial_{t}\left(\varepsilon_{e}\langle\boldsymbol{u}\rangle_{e}\right)=-\rho_{e} \partial_{\boldsymbol{x}} \cdot\left(\varepsilon_{e}\langle\boldsymbol{u}\rangle_{e}\langle\boldsymbol{u}\rangle_{e}\right)-\partial_{\boldsymbol{x}} \cdot\langle\boldsymbol{S}\rangle_{e}-n\langle\boldsymbol{f}\rangle_{p}+\varepsilon_{e} \rho_{e} \boldsymbol{g},
\end{gathered}
$$

where $\varepsilon_{e}$ and $\langle\boldsymbol{u}\rangle_{e}$ are the volume fraction and mean velocity of the fluid, respectively, $\langle\boldsymbol{S}\rangle_{e}$ is the fluid effective stress tensor, $n\langle\boldsymbol{f}\rangle_{p}$ is the mean fluid-particle interaction force, with $n$ denoting the particle number density, and $\boldsymbol{g}$ is the gravitational acceleration. The expression for $\langle\boldsymbol{S}\rangle_{e}$ comes directly from the averaging procedure:

$$
\begin{aligned}
\langle\boldsymbol{S}\rangle_{e}(\boldsymbol{x}, t) \equiv & \varepsilon_{e}(\boldsymbol{x}, t)\langle\boldsymbol{\sigma}\rangle_{e}(\boldsymbol{x}, t)+\varepsilon_{e}(\boldsymbol{x}, t) \rho_{e}\langle\hat{\boldsymbol{u}} \hat{\boldsymbol{u}}\rangle_{e}(\boldsymbol{x}, t)+n(\boldsymbol{x}, t)\langle\boldsymbol{A}\rangle_{p}(\boldsymbol{x}, t) \\
& -(1 / 2) \partial_{\boldsymbol{x}} \cdot\left[n(\boldsymbol{x}, t)\langle\boldsymbol{B}\rangle_{p}(\boldsymbol{x}, t)\right]
\end{aligned}
$$

where:

$$
\begin{gathered}
n(\boldsymbol{x}, t)\langle\boldsymbol{A}\rangle_{p}(\boldsymbol{x}, t) \equiv a \sum_{r}\left[\psi\left(\left|\boldsymbol{x}-z_{r}(t)\right|\right) \int_{\partial \Lambda_{r}} \boldsymbol{k}_{r}(\boldsymbol{z}, t) \boldsymbol{k}_{r}(z, t) \cdot \boldsymbol{\sigma}_{e}(\boldsymbol{z}, t) d \boldsymbol{s}_{z}\right], \\
n(\boldsymbol{x}, t)\langle\boldsymbol{B}\rangle_{p}(\boldsymbol{x}, t) \equiv a^{2} \sum_{r}\left[\psi\left(\left|\boldsymbol{x}-\boldsymbol{z}_{r}(t)\right|\right) \int_{\partial \Lambda_{r}} \boldsymbol{k}_{r}(z, t) \boldsymbol{k}_{r}(z, t) \boldsymbol{k}_{r}(\boldsymbol{z}, t) \cdot \boldsymbol{\sigma}_{e}(\boldsymbol{z}, t) d \boldsymbol{s}_{z}\right] .
\end{gathered}
$$

Here $\langle\boldsymbol{\sigma}\rangle_{e}$ is the volume average of the point stress tensor $\boldsymbol{\sigma}_{e}$ of the fluid, $\langle\hat{\boldsymbol{u}} \hat{\boldsymbol{u}}\rangle_{e}$ is the volume average of the dyadic product of the fluid velocity fluctuations, $\psi$ is the weighting function used in the volume averaging procedure, $z_{r}$ is the (time-dependent) position of the center of the generic particle $r$, and $\boldsymbol{k}_{r}$ is the outward unit normal to the surface $\partial \Lambda_{r}$ bounding particle $r$. The summation is over all the particles in the suspension.

The first term on the right-hand side of Eq. (3) arises because, before the averaging is carried out, the liquid phase is already a fluid —and so it is already endowed with a point stress tensor (no analogous term features in the effective stress tensor of the solid phase). The second term is a Reynolds stress type of contribution. The last two terms are related to the fluid dynamic interaction between the fluid and the particles (through the traction force exerted by the fluid on the particle surfaces). These terms are not solely related to the fluid phase, but involve the particles; for this reason their combined contribution has been referred to as particle-presence stress [30]. Note that we did not choose to ascribe this quantity to the effective stress tensor of the fluid - this is a direct result of volume averaging, a formal mathematical procedure. So the particle-presence stress is not part of the effective stress tensor of the solid phase. Note as well that this conclusion is unaffected by the averaging method adopted; as shown by Zhang and Prosperetti [35], ensemble averaging leads unambiguously to the same conclusion. Many researchers (e.g., Refs. [8,12,15,17,30-33]) have ascribed this contribution to the solid effective stress tensor, an error that often occurs because one misinterprets some terms featuring in the averaged equations of motion (in particular, the average fluid-particle interaction force). This point, clearly raised by Jackson [34], has been recently discussed by Nott et al. [28] (refer also to Appendix A). It is a detail, but it is a crucial one with important consequences. 
Note that, as Eq. (3) reveals, the effective stress tensor of the fluid does not coincide with the average of the point stress tensor of the fluid; that is, $\langle\boldsymbol{S}\rangle_{e} \neq \varepsilon_{e}\langle\boldsymbol{\sigma}\rangle_{e}$. The particle-presence stress is caused by the presence of the particles, but this does not mean that it belongs to the solid effective stress tensor. In Batchelor [36], the particle-presence stress tensor is part of the tensor that Batchelor calls particle stress. But Batchelor never stated that the particle stress tensor coincides with the effective stress tensor of the solid phase; he just grouped in the particle stress tensor all the contributions to the stress tensor of the suspension caused by the particles. In his paper, Batchelor addressed the problem of closure of the stress tensor of the suspension. As discussed in Appendix B, his results are consistent with those reported in Jackson's work. But Batchelor did not address the problem of closure of the effective stress tensors of the fluid and solid phases, and did not discuss to which phasic effective stress tensor the terms included in the suspension stress tensor should belong.

For the solid phase, the mass and linear momentum balance equations read:

$$
\begin{gathered}
\partial_{t} \varepsilon_{s}=-\partial_{\boldsymbol{x}} \cdot\left(\varepsilon_{s}\langle\boldsymbol{u}\rangle_{s}\right), \\
\rho_{s} \partial_{t}\left(\varepsilon_{s}\langle\boldsymbol{u}\rangle_{s}\right)=-\rho_{s} \partial_{\boldsymbol{x}} \cdot\left(\varepsilon_{s}\langle\boldsymbol{u}\rangle_{s}\langle\boldsymbol{u}\rangle_{s}\right)-\partial_{\boldsymbol{x}} \cdot\langle\boldsymbol{S}\rangle_{s}+n\langle\boldsymbol{f}\rangle_{p}+\varepsilon_{s} \rho_{s} \boldsymbol{g},
\end{gathered}
$$

where $\varepsilon_{s}$ and $\langle\boldsymbol{u}\rangle_{s}$ are the volume fraction and mean velocity of the solid, respectively, and $\langle\boldsymbol{S}\rangle_{s}$ is the solid effective stress tensor. This is given by:

$$
\langle\boldsymbol{S}\rangle_{s}(\boldsymbol{x}, t) \equiv \varepsilon_{s}(\boldsymbol{x}, t) \rho_{s}\langle\hat{\boldsymbol{u}} \hat{\boldsymbol{u}}\rangle_{s}(\boldsymbol{x}, t)+n(\boldsymbol{x}, t)\langle\boldsymbol{C}\rangle_{p}(\boldsymbol{x}, t)-(1 / 2) \partial_{\boldsymbol{x}} \cdot\left[n(\boldsymbol{x}, t)\langle\boldsymbol{D}\rangle_{p}(\boldsymbol{x}, t)\right],
$$

with:

$$
\begin{gathered}
n(\boldsymbol{x}, t)\langle\boldsymbol{C}\rangle_{p}(\boldsymbol{x}, t) \equiv a \sum_{r}\left[\psi\left(\left|\boldsymbol{x}-\boldsymbol{z}_{r}(t)\right|\right) \sum_{s \neq r} \boldsymbol{k}_{r s}(t) \boldsymbol{f}_{r s}(t)\right], \\
n(\boldsymbol{x}, t)\langle\boldsymbol{D}\rangle_{p}(\boldsymbol{x}, t) \equiv a^{2} \sum_{r}\left[\psi\left(\left|\boldsymbol{x}-\boldsymbol{z}_{r}(t)\right|\right) \sum_{s \neq r} \boldsymbol{k}_{r s}(t) \boldsymbol{k}_{r s}(t) \boldsymbol{f}_{r s}(t)\right],
\end{gathered}
$$

where $\boldsymbol{f}_{r s}$ is the contact force exerted by particle $r$ on the generic particle $s$ and $\boldsymbol{k}_{r s}$ is the unit vector pointing from the center of particle $r$ to that of particle $s$.

The first contributor to the solid effective stress tensor is referred to as kinetic stress. It is related to the solid velocity fluctuations $\hat{\boldsymbol{u}}_{s}$ and arises from the Reynolds decomposition of the advection term in the solid-phase averaged dynamical equation. The other two contributors constitute the particle-contact stress, which stems from direct particle contacts. These can be of two kinds: nearly instantaneous contacts that form during particle collisions and enduring contacts that establish when suspensions become very dense. The first kind gives rise to the collisional stress and captures the transfer of linear momentum over the distance $2 a$ between the centers of two colliding particles, while the second kind brings about the frictional stress, which, as said, becomes important solely when particles are close to their maximum packing. Note that Eq. (8) does not account for stress induced by interparticle forces (that is, action at a distance forces, such as electrostatic forces). Should these forces be significant, Eq. (8) would have to include a new term, whose expression would be similar to that of the particle-contact stress, provided the interparticle force range is far shorter than the weighting function radius. In this article, we assume that these forces are negligible. Moreover, we focus on (moderately) dense mixtures where the effect of direct particle contacts is insignificant compared to that of fluid-mediated (via lubrication films) particle interactions, our emphasis being on the regions $\mathrm{B}$ and $\mathrm{F}$ of the rheophysical regime classification of Coussot and Ancey [1].

The two-fluid modeling approach has been used to model liquid-particle suspensions. We mention, for instance, the work of Municchi et al. [32], where the fluid effective stress tensor is closed with the Newtonian closure and a constant viscosity, while the solid effective stress tensor comprises two parts, one accounting for the kinetic and collisional stresses, closed via the kinetic theory model of Gidaspow [18], and one accounting for the stress anisotropy (i.e., the normal stress differences), 
expressed via the closure of Morris and Boulay. Hence, in this paper the particle-presence stress is ascribed to the solid effective stress tensor, a choice that, as we discussed, we believe is incorrect. Also, the part of the particle-presence stress tensor resulting in the viscosity correction of Einstein [38] seems to be missing, since it can neither arise from the kinetic and collisional stresses nor from the anisotropic stress.

Another interesting work based on the two-fluid modeling approach is that of Drijer et al. [39]. Here particle migration is modeled through a force fulfilling the action and reaction principle (a force present in the dynamical equations of both phases with opposite signs). This is close to what we will suggest at the end of this article. The difference is in how the force is modelled: Drijer et al. use the diffusion-flux model, while we relate it to the particle-presence stress tensor.

\section{B. The mixture model}

The mixture model treats the suspension as an effective fluid. For its derivation, we refer to the literature (for instance, see Ref. [14]). The mass and linear momentum balance equations for the mixture read:

$$
\begin{gathered}
\partial_{t} \rho_{m}=-\partial_{\boldsymbol{x}} \cdot\left(\rho_{m}\langle\boldsymbol{u}\rangle_{m}\right) \\
\partial_{t}\left(\rho_{m}\langle\boldsymbol{u}\rangle_{m}\right)=-\partial_{\boldsymbol{x}} \cdot\left(\rho_{m}\langle\boldsymbol{u}\rangle_{m}\langle\boldsymbol{u}\rangle_{m}\right)-\partial_{\boldsymbol{x}} \cdot\langle\boldsymbol{S}\rangle_{m}+\rho_{m} \boldsymbol{g},
\end{gathered}
$$

where:

$$
\rho_{m} \equiv \varepsilon_{e} \rho_{e}+\varepsilon_{s} \rho_{s} ; \quad \rho_{m}\langle\boldsymbol{u}\rangle_{m} \equiv \varepsilon_{e} \rho_{e}\langle\boldsymbol{u}\rangle_{e}+\varepsilon_{s} \rho_{s}\langle\boldsymbol{u}\rangle_{s}
$$

The effective stress tensor of the mixture, denoted by $\langle\boldsymbol{S}\rangle_{m}$, comprises three contributions:

$$
\langle\boldsymbol{S}\rangle_{m} \equiv\langle\boldsymbol{S}\rangle_{e}+\langle\boldsymbol{S}\rangle_{s}+\langle\boldsymbol{S}\rangle_{d}
$$

$\langle\boldsymbol{S}\rangle_{d}$ is called diffusion stress tensor [40] and arises because each phase moves at a velocity that differs from that of the mixture. It is defined as follows:

$$
\langle\boldsymbol{S}\rangle_{d} \equiv \rho_{m} \omega_{e} \omega_{s}\left(\langle\boldsymbol{u}\rangle_{s}-\langle\boldsymbol{u}\rangle_{e}\right)\left(\langle\boldsymbol{u}\rangle_{s}-\langle\boldsymbol{u}\rangle_{e}\right),
$$

where $\omega_{e}$ and $\omega_{s}$ are the mass fractions of the fluid and solid phases, respectively. Its effect is usually negligible compared to that of the phasic effective stress tensors.

In addition to the continuity equation for the suspension, another mass balance equation is necessary to track the volume fractions of the phases. Usually, this equation is written for the solid phase and reads:

$$
\partial_{t} \varepsilon_{s}=-\partial_{\boldsymbol{x}} \cdot\left(\varepsilon_{s}\langle\boldsymbol{u}\rangle_{m}\right)-\partial_{\boldsymbol{x}} \cdot\left[\varepsilon_{s} \omega_{e}\left(\langle\boldsymbol{u}\rangle_{s}-\langle\boldsymbol{u}\rangle_{e}\right)\right] .
$$

The term in square brackets on the right-hand side of this equation arises because the solid does not move at the same velocity as the mixture. This term can be interpreted as a diffusive flux, but it does not usually obey Fick's law.

As shown, diffusive fluxes (of mass and linear momentum) arise in Eqs. (12) and (16). These terms are unclosed, because instead of the suspension velocity, they involve the fluid-particle slip velocity. This velocity calls for an additional (dynamical) equation. If the fluid and solid velocity fields relax to local equilibrium rapidly, it is:

$$
\langle\boldsymbol{u}\rangle_{s}-\langle\boldsymbol{u}\rangle_{e}=-(1 / \beta)\left[\varepsilon_{e} \partial_{\boldsymbol{x}} \cdot\langle\boldsymbol{S}\rangle_{s}-\varepsilon_{s}\left(\rho_{s}-\rho_{m}\right)\left(\boldsymbol{g}-D_{t}\langle\boldsymbol{u}\rangle_{m}\right)\right] .
$$

This equation is approximate and is based on an asymptotic expansion on the particle Stokes number, which, for the systems of interest here, is assumed to be much smaller than unity; for details, we refer to the literature (for instance, see Refs. [13,14,40]).

In Eq. (17), $D_{t}\langle\boldsymbol{u}\rangle_{m}$ represents the substantial derivative of the mixture velocity field and $\beta$ is the drag force coefficient, for which many closures are available in the literature $[13,41,42]$. Here we 
report that of Wen and Yu [43]:

$$
\beta=\frac{3}{4} C_{D}(\operatorname{Re}) \frac{\rho_{e} \varepsilon_{s}\left|\langle\boldsymbol{u}\rangle_{s}-\langle\boldsymbol{u}\rangle_{e}\right|}{2 a} \varepsilon_{e}^{-1.65}
$$

where:

$$
\operatorname{Re} \equiv \frac{\rho_{e} \varepsilon_{e}\left|\langle\boldsymbol{u}\rangle_{s}-\langle\boldsymbol{u}\rangle_{e}\right|(2 a)}{\mu_{e}}
$$

and

$$
C_{D}(\operatorname{Re})=\left\{\begin{array}{ll}
(24 / \mathrm{Re})\left(1+0.15 \mathrm{Re}^{0.687}\right) & \text { for } \mathrm{Re}<1000 \\
0.44 & \text { for } \operatorname{Re} \geqslant 1000
\end{array} .\right.
$$

Here Re is the particle Reynolds number. Under the conditions in which the mixture model holds, the fluid and solid mean velocities are equal at leading order in the Stokes number, and the slip velocity vanishes; thus, $\operatorname{Re} \lll 1, C_{D}(\operatorname{Re}) \rightarrow 24 / \mathrm{Re}$ and for neutrally buoyant particles of constant density dispersed in a fluid of constant viscosity, Eq. (16) reduces to:

$$
\partial_{t} \varepsilon_{s}=-\partial_{\boldsymbol{x}} \cdot\left(\varepsilon_{s}\langle\boldsymbol{u}\rangle_{m}\right)-2 a^{2} /\left(9 \mu_{e}\right) \partial_{\boldsymbol{x}} \cdot\left(\varepsilon_{e}^{4.65} \partial_{\boldsymbol{x}} \cdot\langle\boldsymbol{S}\rangle_{s}\right) .
$$

This is the equation usually used to describe shear-induced particle migration. As discussed in Sec. I, this equation suggests that neutrally buoyant particles migrate toward regions of low shear rate only if $\langle\boldsymbol{S}\rangle_{s}$ is nonuniform.

\section{CLOSURES FOR THE SOLID EFFECTIVE STRESS TENSOR}

Equation (17) indicates that particle migration can be induced by gravity, inertia, and gradients in the solid effective stress tensor. For neutrally buoyant particles, the first two have no effect; if we trust Eq. (17), then we conclude that particle migration is driven solely by gradients in $\langle\boldsymbol{S}\rangle_{s}$. Hence, the key to describing particle migration correctly is having an accurate closure for this tensor, a closure that accounts accurately for all the phenomena generating stress in the solid phase. As Eq. (8) reveals, several phenomena contribute: particle velocity fluctuations (which generate kinetic stress), particle collisions and/or enduring particle contacts (which together generate particle-contact stress), and, if present, interparticle forces. We have assumed that the effects of the last three are negligible. This is not always true, but experimental evidence indicates that migration starts to be appreciable in moderately dense mixtures falling in the region B of the rheophysical regime classification of Coussot and Ancey [1], where these three contributions are negligible. We investigate the cause of migration in these conditions, where particle-contact and interparticle-force stresses can be ruled out, because we want to focus on the role played by the kinetic stress. Our analysis will show that this part of the solid effective stress tensor cannot be the main driver of particle migration, a result revealing that migration must have fluid dynamic origin. Assuming that migration is induced by the lubrication forces between particles, we will then prove that the migration velocity is related to the gradients in the particle-presence stress tensor associated with such forces. Since lubrication forces, direct particle contact forces, and interparticle forces are all pointwise and pairwise, extending our results to cases where the last two forces are present would be straightforward.

\section{A. The closure of Buyevich}

For the systems of interest in our study, it seems that particle migration must be driven by the kinetic stress induced by particle velocity fluctuations. Buyevich [3] agreed with this idea. When studying particle migration, he thus focused on developing a constitutive equation for $\langle\boldsymbol{S}\rangle_{s}$ accounting solely for the kinetic stress. He argued that in shear flows this originates from the fluid-mediated interactions between particles when adjacent mixture layers move past one another. These interactions yield random particle displacements of the order of the particle size, leading 
to "shear-induced" particle velocity fluctuations. With heuristic arguments based on this idea, he proposed the following closure:

$$
\langle\boldsymbol{S}\rangle_{s}=C \rho_{s} a^{2} \varphi\left(\varepsilon_{s}\right)\left[\left(\pi^{2} / 4-1\right)\langle\dot{\boldsymbol{\gamma}}\rangle_{s} \cdot\langle\dot{\boldsymbol{\gamma}}\rangle_{s}+\left|\langle\dot{\boldsymbol{\gamma}}\rangle_{s}\right|^{2} \boldsymbol{I}\right] .
$$

Here $C$ is an unknown coefficient. Because the closure was developed with scaling arguments, this coefficient was expected to be of unit order of magnitude. But later, by fitting the model results to experimental data for a two-dimensional channel flow, Buyevich and Kapbsov [29] suggested the value of 0.016 . In Eq. (22), $\langle\dot{\gamma}\rangle_{s}$ is twice the deformation rate tensor of the solid mean velocity field, and $\left|\langle\dot{\gamma}\rangle_{s}\right|$ is its magnitude; these quantities can be replaced with the equivalent ones for the mixture velocity field (i.e., $\langle\dot{\gamma}\rangle_{m}$ and $\left|\langle\dot{\gamma}\rangle_{m}\right|$ ) inasmuch as at leading order the two velocity fields are equal. Finally, it is:

$$
\varphi\left(\varepsilon_{s}\right)=\varepsilon_{s}^{3}\left[\frac{1+\varepsilon_{s}+\varepsilon_{s}^{2}-\varepsilon_{s}^{3}}{\left(1-\varepsilon_{s}\right)^{3}}+2.9 \frac{\left(\varepsilon_{s} / \varepsilon_{s}^{\star}\right)^{3}}{1-\left(\varepsilon_{s} / \varepsilon_{s}^{\star}\right)}\right]\left[\frac{1-0.5 \varepsilon_{s}}{\left(1-\varepsilon_{s}\right)^{3}}+1.08 \frac{\left(\varepsilon_{s} / \varepsilon_{s}^{\star}\right)^{3}}{1-\left(\varepsilon_{s} / \varepsilon_{s}^{\star}\right)}\right]^{2},
$$

where $\varepsilon_{s}^{\star}$ is the maximum value that the solid volume fraction can attain when the suspension reaches packing conditions. This value is often taken to be (around) 0.65 .

Equation (22) predicts the fully developed solid volume fraction profiles in wide-gap Couette flows and pressure-driven laminar pipe flows fairly well [3,29]; notwithstanding, it does present two problems, which have not been reported in the literature.

The first is related to the timescale of the migration process predicted by this equation. To investigate this problem, we considered a wide-gap Couette flow of a dense suspension of neutrally buoyant particles between two vertical concentric cylinders, the inner (radius $\kappa R$ ) rotating with angular velocity $\Omega$ around its axis and the outer (radius $R$ ) being motionless. The flow is laminar, and we may take the mixture velocity field to be tangent to the angular coordinate lines. If we also assume cylindrical symmetry and neglect end effects, then we can take the velocity and the solid volume fraction fields to depend only on the radial coordinate (and, for transient flows, on the time). With these assumptions, adopted by most of the researchers modeling this flow, the first term on the right-hand side of Eq. (21) vanishes (that is, convection plays no role) and the equation reduces to:

$$
\partial_{t} \varepsilon_{s}=-2 a^{2} /\left(9 \mu_{e}\right) \partial_{r}\left\{r \varepsilon_{e}^{4.65}\left[\partial_{r}\langle S\rangle_{s, r r}+(1 / r)\left(\langle S\rangle_{s, r r}-\langle S\rangle_{s, \theta \theta}\right)\right]\right\},
$$

where $\langle S\rangle_{s, r}$ and $\langle S\rangle_{s, \theta \theta}$ denote the $r r$ and $\theta \theta$ components of the tensor $\langle\boldsymbol{S}\rangle_{s}$. We solved this equation numerically, using Eqs. (22) and (23) to close $\langle\boldsymbol{S}\rangle_{s}$.

The evolution of the solid volume fraction profile in this flow was obtained experimentally by Phillips et al. [22]. In their setup $R=2.38 \mathrm{~cm}$ and $\kappa=0.27$, and the suspension consisted of polymethylmethacrylate particles with density $\rho_{s}=1182 \mathrm{~kg} / \mathrm{m}^{3}$ dispersed in a liquid with the same density and viscosity $\mu_{e}=4.95 \mathrm{Pas}$. The mean diameter of the particles was either 100 or $675 \mu \mathrm{m}$. The suspension was initially homogeneous with a solid volume fraction $\bar{\varepsilon}_{s}$ of either 0.50 or 0.55 .

Figure 1(a) reports the solid volume fraction profile, obtained from the solution of Eq. (24) for a mixture with $2 a=675 \mu \mathrm{m}$ and $\bar{\varepsilon}_{s}=0.5$ and the inner cylinder rotating at $\Omega=17 \mathrm{rmp}$, after $470 \mathrm{~min}$ from the start of rotation, a time equivalent to 8000 revolutions of the inner cylinder. As we can see, if in Eq. (22) we set $C=1$ (the order of magnitude that Buyevich [3] initially expected for $C$ ), then the model predicts very weak migration, the solid volume fraction remaining uniform over most of the flow domain (except close to the inner wall). Setting $C=0.016$, as suggested by Buyevich and Kapbsov [29], predicts even weaker migration, because smaller values of $C$ result in smaller migration fluxes. Equation (22) fails to capture correctly the timescale of migration, unless the value of $C$ is increased significantly. As Fig. 1(a) shows, for $C=10^{3}$ the numerical results do match the experimental data sufficiently well, but such a high value for $C$ is physically unjustified.

Figure 1(b) reports the solid volume fraction profiles for the same system, but with $\bar{\varepsilon}_{s}=0.55$, after 200, 800, and 12000 revolutions of the inner cylinder. The numerical results, obtained for $C=10^{3}$, again match the experimental data quite well, an outcome that appears to confirm the higher order of magnitude found for the coefficient $C$. 

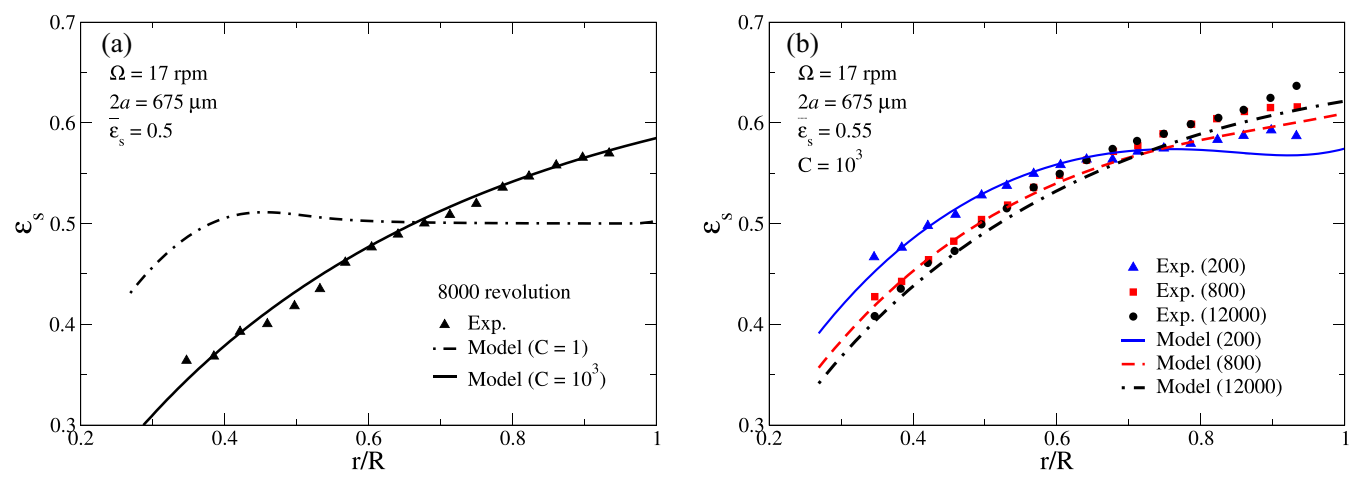

FIG. 1. Solid volume fraction profiles in a wide-gap Couette flow. The outer cylinder is stationary, while the inner one rotates at angular velocity $\Omega$. The suspensions, of neutrally buoyant particles with mean radius $a$, are initially uniform with solid volume fraction $\bar{\varepsilon}_{s}$. Model curves from Eqs. (22) and (24). Experimental data from Phillips et al. [22].

But when this larger value of $C$ is used to predict the migration of particles with a mean diameter of $100 \mu \mathrm{m}$, the model fails to reproduce the experimental data, again predicting very weak migration and a solid volume fraction profile uniform over most of the flow domain. In this case, as Fig. 2 shows, the value required for a good match is $C=4.5 \times 10^{4}$. As $4.5 \times 10^{4} / 10^{3}$ is nearly equal to $(675 / 100)^{2}$, this suggest that $C \propto 1 / a^{2}$, a dependence that is inconsistent with the derivation of Eq. (22), where $C$ must be a constant (that is, its value cannot depend on the properties of the suspension). Note that in Couette flows the steady-state particle volume fraction profile does not depend on the coefficient $C$ [3], and so the issue just discussed did not arise when Buyevich tested his closure on these stationary flows.

The second problem posed by Eq. (22) is about the incorrect direction of the migration flux in parallel-plate rheometers. Consider a dense suspension in one of such rheometers (plates in the $r, \theta$ plane) with a gap size $H$ (in the $z$ direction). The bottom plate is stationary, and the top one

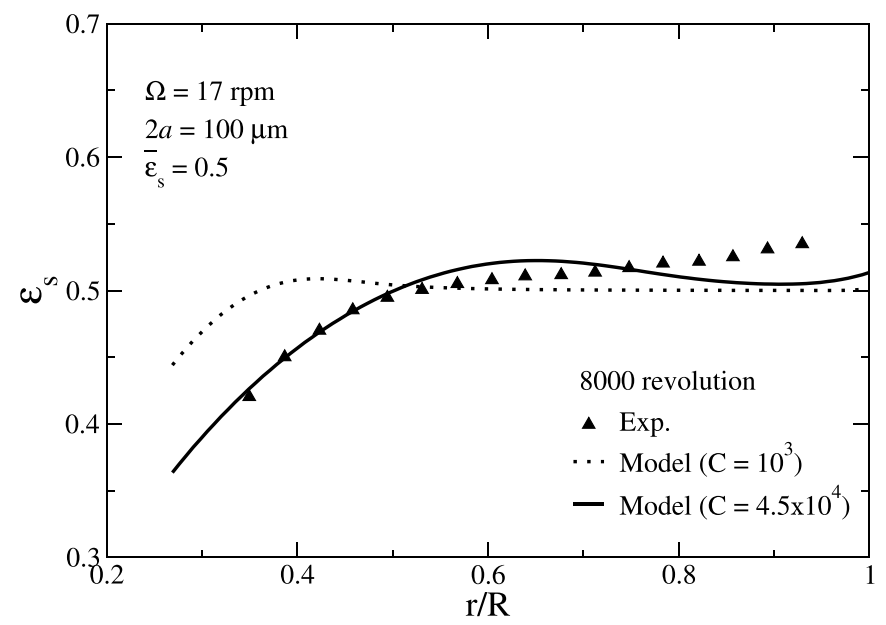

FIG. 2. Solid volume fraction profiles in a wide-gap Couette flow. The outer cylinder is stationary, while the inner one rotates at angular velocity $\Omega$. The suspensions, of neutrally buoyant particles with mean radius $a$, are initially uniform with solid volume fraction $\bar{\varepsilon}_{s}$. Model curves from Eqs. (22) and (24). Experimental data from Phillips et al. [22]. 


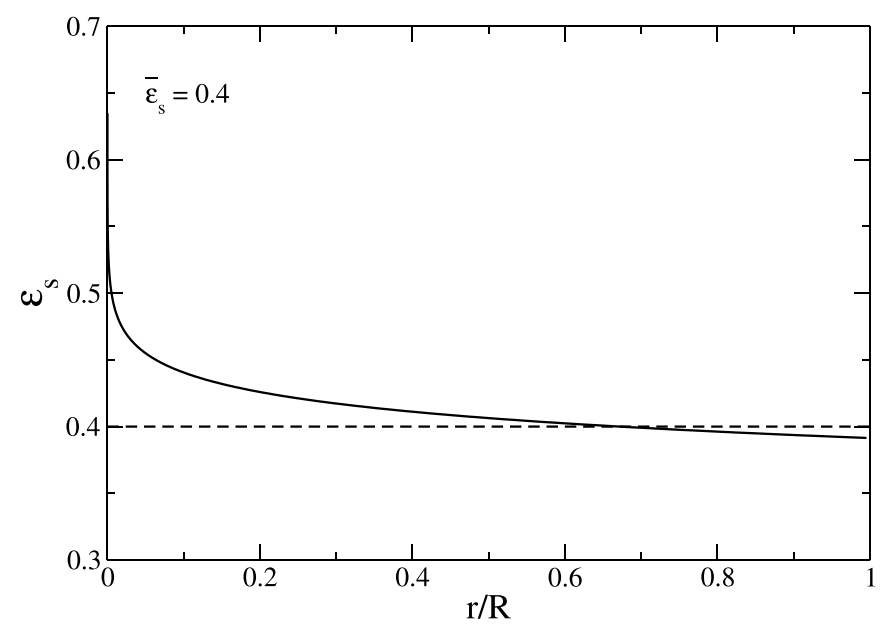

FIG. 3. Steady-state solid volume fraction profile in a plate-plate rheometer for a dense suspension with initial homogenous concentration $\bar{\varepsilon}_{s}=0.4$ obtained using Eq. (25) and by assuming $\varepsilon_{s}^{\star}=0.68$. This equation predicts an inward flux of particles that contradicts experimental observations. The horizontal line at $\varepsilon_{s}=0.4$ is plotted to guide the eye.

rotates with angular velocity $\Omega$ around the $z$ axis. Because at leading order the mean velocities of the solid and the mixture are equal, we can assume $\langle\dot{\gamma}\rangle_{s}=\langle\dot{\gamma}\rangle_{m}$. Also, neglecting the secondary flow in the rheometer, we may take $\langle u\rangle_{m, r}=\langle u\rangle_{m, z}=0$ and $\langle u\rangle_{m, \theta}=\Omega r z / H$ (in Sec. III C, using scaling analysis, we prove the effects of secondary flow are negligible). Using these assumptions, we can obtain the stress tensor of the solid phase from Eq. (22) and use it to solve Eq. (24) in steady-state conditions. This leads to the following equation, which yields the radial profile of the solid volume fraction:

$$
\frac{d \varepsilon_{s}}{d r}=\frac{\pi^{2}-12}{4 r} \frac{\varphi\left(\varepsilon_{s}\right)}{d \varphi\left(\varepsilon_{s}\right) / d \varepsilon_{s}} \quad \text { with } \quad \frac{2}{R} \int_{0}^{R} \varepsilon_{s}(r) r d r=\bar{\varepsilon}_{s} .
$$

The condition on the right ensures the total mass of solid is conserved. Here $R$ is the radius of the plates in the rheometer, and $\bar{\varepsilon}_{s}$ is the initial bulk solid concentration.

Since $\varphi\left(\varepsilon_{s}\right)$ is always positive and increases monotonically with $\varepsilon_{s}$, diverging for $\varepsilon_{s} \rightarrow \varepsilon_{s}^{\star}$, we conclude that $d \varepsilon_{s} / d r<0$. This implies that the particles migrate toward the rotation axis (inward migration). As an example, Fig. 3 reports the solid volume fraction profile obtained from Eq. (25) for a suspension that is initially uniform, with $\bar{\varepsilon}_{s}=0.4$ (and $\varepsilon_{s}^{\star}=0.68$ ). As we see, for $r \rightarrow 0, \varepsilon_{s} \rightarrow \varepsilon_{s}^{\star}$, since $d \varepsilon_{s} / d r \rightarrow \infty$. This steady-state result is independent of the value of the coefficient $C$, the closure adopted for the drag coefficient, and the fluid and solid physical properties. The inward flux predicted by Eq. (25), similarly to that obtained from the diffusive-flux model, is incorrect, because experiments show that in this setup particles either do not migrate $[23,24]$ or migrate away from the rotation axis $[7,25,26]$.

In light of these results, we conclude that either Eq. (22) is incorrect or the kinetic stress is not the main driver of particle migration. As the arguments of Buyevich [3] in his derivation appear to be sound and compelling, we favor the second explanation. This is in line with the idea proposed by Lhuillier [44] and followed by Nott et al. [28].

We conclude with the following consideration. The study just conducted reveals that the kinetic stress cannot be the main driver of migration. This suggests that migration must have fluid dynamic origin. If we require that the order of magnitude of $\langle\boldsymbol{S}\rangle_{s}$ in Eq. (22) be equal to $\varphi\left(\varepsilon_{s}\right) \mu_{e}\left|\langle\dot{\boldsymbol{\gamma}}\rangle_{s}\right|$, which is expected to estimate well the order of magnitude of the viscous stress in the suspension, we 
obtain:

$$
C \rho_{s} a^{2} \varphi\left(\varepsilon_{s}\right)\left|\langle\dot{\gamma}\rangle_{s}\right|^{2} \sim \varphi\left(\varepsilon_{s}\right) \mu_{e}\left|\langle\dot{\gamma}\rangle_{s}\right|,
$$

whence:

$$
C \sim \frac{\mu_{e}}{\rho_{s} a^{2}\left|\langle\dot{\gamma}\rangle_{s}\right|} \sim \frac{\mu_{e}}{\rho_{e} a^{2}\left|\langle\dot{\gamma}\rangle_{s}\right|} \sim\left(\frac{L}{a}\right)^{2}\left(\frac{\mu_{e}}{\rho_{e} U L}\right) \equiv\left(\frac{L}{a}\right)^{2} \frac{1}{\operatorname{Re}_{L}},
$$

where $U$ and $L$ are the velocity and length scales of the flow of the suspension. This result confirms our previous finding: $C \propto 1 / a^{2}$. It also indicates that the time required by the particle kinetic stress to induce significant particle migration exceeds considerably the timescale of the migration process, the ratio between these two timescales being proportional to the reciprocal of the Reynolds number. For vanishingly small values of the Reynolds number, particle velocity fluctuations clearly cannot be responsible for particle migration.

\section{B. The closure of Morris and Boulay}

The problem of closure for the solid effective stress tensor was addressed heuristically by Morris and Boulay [15], who aimed to investigate the effect of normal stresses in concentrated mixtures on shear-induced particle migration. Assuming that the dominant stresses originate from fluid dynamic lubrication forces between particle pairs (and contact forces, which for the systems of interest in our work are negligible), Morris and Boulay suggested the following constitutive equation:

$$
\langle\boldsymbol{S}\rangle_{s}=-\eta_{p}\left(\varepsilon_{s}\right)\langle\dot{\boldsymbol{\gamma}}\rangle_{m}+\eta_{n}\left(\varepsilon_{s}\right)\left|\langle\dot{\boldsymbol{\gamma}}\rangle_{m}\right| \boldsymbol{Q} .
$$

The first term on the right is the Newtonian part of the solid stress tensor; $\eta_{p}\left(\varepsilon_{s}\right)$ represents the particle contribution to the suspension shear viscosity (in the dilute limit, this coincides with the Einstein correction to the mixture viscosity [38]). The second term accounts for the presence of viscous normal stresses; $\eta_{n}\left(\varepsilon_{s}\right)$ is the normal stress viscosity, and $\boldsymbol{Q}$ is a diagonal, anisotropic material property tensor featuring two fitting parameters.

Morris and Boulay showed that if these parameters are properly tuned, then Eq. (28) predicts well steady-state solid volume fraction profiles in a number of viscometric flows and transient solid volume fraction profiles in wide-gap Couette flows. Nevertheless, their closure presents a conceptual problem: It ascribes the particle-presence stress to the solid phase.

As discussed in Sec. II, the particle-presence stress tensor [the sum of the terms in Eqs. (4) and (5)] is part of the fluid effective stress tensor - this being a direct result of the averaging procedure used to derive the multifluid equations of change (see, for instance, Refs. [14,28,34,45]). The particle-presence stress tensor captures the contribution of the lubrication films between particle pairs; this contribution, therefore, belongs to the fluid effective stress tensor, $\langle\boldsymbol{S}\rangle_{e}$ (an aspect discussed at length in Nott et al. [28] and Jamshidi et al. [14]). Hence, it seems Eq. (28) does pose a problem. But the numerical results of Morris and Boulay agree with experimental data. How can this be explained?

In their calculations, it appears that the authors did not use the correct expression for $\eta_{p}$. In Eq. (28), instead of using $\eta_{p}$ (the particle contribution to the mixture shear viscosity), they used the mixture shear viscosity (equal to $\eta_{p}$ plus the shear viscosity of the ambient fluid, $\mu_{e}$ ). Hence, they did not employ the closure they had proposed. Moreover, they solved the linear momentum balance equation for the solid phase only, without considering the corresponding equation for the fluid phase. For instance, consider the steady wide-gap Couette flow, studied in Sec. III B of Ref. [15]. Because the flow is steady and laminar, and the particles are neutrally buoyant, the following dynamical equations hold:

$$
\begin{aligned}
& \partial_{\boldsymbol{x}} \cdot\langle\boldsymbol{S}\rangle_{e}=\rho_{m} \boldsymbol{g} \\
& \partial_{\boldsymbol{x}} \cdot\langle\boldsymbol{S}\rangle_{s}=\mathbf{0}
\end{aligned}
$$


The divergence of the solid effective stress tensor vanishes insofar as in Eq. (17) the slip velocity (on the left) and the inertial and gravitational terms (on the right) are zero. In the "angular direction," Eq. (29a) yields:

$$
\left(\partial_{\boldsymbol{x}} \cdot\langle\boldsymbol{S}\rangle_{e}\right)_{\theta}=\left(1 / r^{2}\right) \partial_{r}\left(r^{2}\langle S\rangle_{e, r \theta}\right)+(1 / r) \partial_{\theta}\langle S\rangle_{e, \theta \theta}+\partial_{z}\langle S\rangle_{e, z \theta}=0 .
$$

An identical relation holds for the effective stress tensor of the solid phase. Then, eliminating the terms that vanish, we obtain:

$$
\begin{aligned}
& \frac{d\left(r^{2}\langle S\rangle_{e, r \theta}\right)}{d r}=0 ; \\
& \frac{d\left(r^{2}\langle S\rangle_{s, r \theta}\right)}{d r}=0 .
\end{aligned}
$$

Morris and Boulay solved only the equation for the solid (that is, the second equation above). Here we shall consider also the equation for the fluid, since both equations must be satisfied. We now use Eq. (28) to express the solid effective stress tensor (considering, as we should, the shear viscosity owing to the particles presence only). With Morris and Boulay, we assume the effective shear viscosity of the fluid coincides with the shear viscosity of the ambient fluid (an expression which is at variance with the analyses of Jackson [34] and Zhang and Prosperetti [35], because in these works the shear viscosity due to the particles presence contributes to the effective viscosity of the fluid; this is again related to the particle-presence stress tensor and its being part of $\langle\boldsymbol{S}\rangle_{e}$ ). Therefore, we write:

$$
\begin{aligned}
& \langle S\rangle_{e, r \theta}=-\mu_{e}\langle\dot{\gamma}\rangle_{m} ; \\
& \langle S\rangle_{s, r \theta}=-\eta_{p}\left(\varepsilon_{s}\right)\langle\dot{\gamma}\rangle_{m},
\end{aligned}
$$

where $\langle\dot{\gamma}\rangle_{m}$ is the shear rate. Being $\mu_{e}$ a constant, Eq. (31a) yields $d\left(r^{2}\langle\dot{\gamma}\rangle_{m}\right) / d r=0$. Equation (31b) consequently yields:

$$
\eta_{p}\left(\varepsilon_{s}\right) \frac{d\left(r^{2}\langle\dot{\gamma}\rangle_{m}\right)}{d r}+r^{2}\langle\dot{\gamma}\rangle_{m} \frac{d \eta_{p}\left(\varepsilon_{s}\right)}{d r}=r^{2}\langle\dot{\gamma}\rangle_{m} \frac{d \eta_{p}\left(\varepsilon_{s}\right)}{d r}=0
$$

whence:

$$
\eta_{p}\left(\varepsilon_{s}\right)=\text { const. }
$$

This means that at steady-state the solid volume fraction is uniform, so that no migration has occurred, a result that is incorrect.

Morris and Boulay [15] did find a good match between numerical results and experimental data, but this is because in Eq. (32b) they inadvertently replaced $\eta_{p}$ with $\eta_{s} \equiv \mu_{e}+\eta_{p}$ (in Ref. [15], see the untagged equation after Eq. (28); notice that there $\eta_{s}$ denotes the shear viscosity of the mixture) and the only equation they solved is as follows:

$$
\partial_{\boldsymbol{x}} \cdot\left[\eta_{s}\left(\varepsilon_{s}\right)\langle\dot{\boldsymbol{\gamma}}\rangle_{m}-\eta_{n}\left(\varepsilon_{s}\right)\left|\langle\dot{\boldsymbol{\gamma}}\rangle_{m}\right| \boldsymbol{Q}\right]=\mathbf{0} .
$$

In the work of Morris and Boulay, the results of this equation matched well the experimental data. In Sec. IV, we shall show why this equation is sound. More details about the above considerations are given in Appendix C, where, to avoid any misunderstandings, we employed the notation of Morris and Boulay [15].

\section{Secondary flow effects}

To conclude this analysis, we investigate the competition between the particle segregation induced by shear-induced particle migration and the particle mixing induced by convection. We focus on parallel-plate rheometers. In them, mixing is caused by the secondary flow due to inertia, for the primary flow does not move particles radially via convection. 
In this type of rheometer, experiments reveal that either no migration [23,24] or outward migration [7,25,26] occurs. To match the experimental data, Morris and Boulay [15] tuned the fitting parameters in Eq. (28), neglecting the contribution of the secondary flow. Doing this is impossible with the closure of Buyevich [3], for it always predicts inward particle migration. Buyevich maintained that the wrong direction of the migration flux did not imply his closure was incorrect. He argued that the secondary flow mixes the particles, counteracting the effect of shear-induced particle migration. The net result is no particle migration. In other words, Buyevich claimed that the convective mixing induced by the secondary flow erases the effect of shear-induced particle migration keeping the solid concentration uniform.

To test Buyevich's argument, we use scaling analysis, comparing the timescales of the two processes. To estimate the timescale of shear-induced particle migration, we avoid Eq. (22), because, with the original value of the coefficient $C$, it predicts the migration time incorrectly. Instead, we employ the constitutive equation in Eq. (35). At leading order, the radial and axial velocity components are zero and $\langle u\rangle_{m, \theta}=\Omega r z / H$, so that $\left|\left\langle\dot{\gamma}_{m}\right\rangle\right|=\Omega r / H$. Then, from Eqs. (16) and (17), the migration timescale results to be

$$
\tau_{M} \sim \frac{R}{\omega_{e}\left(\langle u\rangle_{s, r}-\langle u\rangle_{e, r}\right)} \sim\left(\frac{9 \mu_{e} \varepsilon_{s}}{2 a^{2} \varepsilon_{e}^{4.65}}\right) \frac{H R}{\eta_{n} \Omega} .
$$

To estimate the timescale of the mixing process caused by the secondary flow, we employ the expression for the secondary-flow radial velocity derived by Savins and Metzner [46] (see also Ref. [25]), which holds for parallel-plate rheometers with $H / R \lll 1$. This yields:

$$
\tau_{C} \sim \frac{R}{\langle u\rangle_{m, r}} \sim \frac{100 \eta_{m}}{\rho_{m}(\Omega H)^{2}} .
$$

For a mixture of neutrally buoyant particles with $a=100 \mu \mathrm{m}, \rho_{m}=1000 \mathrm{~kg} / \mathrm{m}^{3}, \mu_{e}=1 \mathrm{Pas}$, $\varepsilon_{s}=0.40$ and $\varepsilon_{s}^{\star}=0.65$ in a rheometer with $R=1 \mathrm{~cm}$ and $H=1 \mathrm{~mm}$, with a plate rotating at the typical angular velocity $\Omega=0.1 \mathrm{rad} / \mathrm{s}$, if to obtain the normal shear viscosity and the mixture shear viscosity we employ the expressions suggested by Morris and Boulay [15] (not shown for brevity), we obtain $\tau_{M} \sim 10^{5} \mathrm{~s}$ and $\tau_{C} \sim 10^{8} \mathrm{~s}$. Consequently, the mixing caused by the secondary flow cannot counterbalance the segregation induced by shear-induced particle migration. Buyevich's argument seems not to stand.

\section{Summary}

We have investigated two well-known closures for the solid effective stress tensor. That of Buyevich [3] is based on the assumption that the solid stress stems from the particle velocity fluctuations generated by particle interactions occurring in shear flow when adjacent mixture layers move past one another. The closure was derived heuristically, but on solid arguments, and predicted well the fully developed solid volume fraction profile in a number of viscometric flows. But it had not been used to predict the time evolution of solid volume fraction profiles. Our analysis revealed two shortcomings of the Buyevich closure: It predicts the timescale of shear-induced particle migration incorrectly, and in parallel-plate rheometers, it predicts the migration direction incorrectly. The argument put forward by Buyevich to justify the second shortcoming, related to secondary-flow convective mixing, seems inadequate. These findings, in our opinion, do not mean that Buyevich's closure is incorrect: They mean that kinetic stress is not the main driver of shear-induced particle migration.

Morris and Boulay [15] agreed with this viewpoint. They believed the driver of the process was the particle-presence stress-in particular the part stemming from the lubrication forces between particle pairs (we are referring to systems where particle collisions, enduring particle contacts and interparticle forces are absent or negligible). Accordingly, the driver has viscous nature. As pointed out by Nott et al. [28] (and more recently in Ref. [14]), this closure presents a problem on theoretical grounds, since the particle-presence stress contributes to the effective stress tensor of the fluid phase, 
while the shear-induced particle migration is related to the nonuniformity of the effective stress tensor of the solid phase [see Eq. (17)]. Notwithstanding, in the form given in Eq. (35), the closure does yield good results. This outcome has to be investigated further.

\section{THE ROLE OF THE FLUID-PARTICLE INTERACTION FORCE IN SHEAR-INDUCED PARTICLE MIGRATION}

As said before, our analysis is restricted to suspensions where the effect of direct particle contacts is insignificant compared with that of fluid-mediated (via lubrication films) particle interactions. In other words, the particle-contact stress (a contributor to the effective stress of the solid) is negligible. In the discussion above, we concluded that the kinetic stress in the solid phase (the only other contributor to the effective stress of the solid) is also negligible in promoting shear-induced particle migration. So, in Eq. (17), the term related to $\langle\boldsymbol{S}\rangle_{s}$ appears to be insignificant. But for suspensions of neutrally buoyant particles this is the only term on the right-hand side of the equation that is nonzero. If we neglect it, the migration velocity vanishes. It seems, therefore, that in Eq. (17) the term representing the main driver of particle migration is missing. This is the conclusion reached by Nott et al. [28], who after proposed a convincing solution to this hurdle. Their idea is that migration is driven by the part of the fluid-particle interaction force arising from the lubrication films between the particles (we refer to Ref. [28] for further details). Building on this notion, we now show that this force is closely related to the particle-presence stress- the very term that Morris and Boulay [15] had identified as the main driver of migration. In the end, our analysis will explain why Eq. (35) did predict steady solid volume fraction profiles correctly.

The starting point in our analysis is the expression that volume averaging provides for the fluidparticle interaction force [34]. This reads:

$$
n(\boldsymbol{x}, t)\langle\boldsymbol{f}\rangle_{p}(\boldsymbol{x}, t) \equiv-\sum_{r}\left[\psi\left(\left|\boldsymbol{x}-\boldsymbol{z}_{r}(t)\right|\right) \int_{\partial \Lambda_{r}} \boldsymbol{k}_{r}(z, t) \cdot \boldsymbol{\sigma}_{e}(z, t) d \boldsymbol{s}_{z}\right]
$$

in which $\sigma_{e}$ is the point stress tensor of the fluid, $\psi$ is the weighting function employed in the volume-averaging procedure, $z_{r}$ is the position of the center of the generic particle $r$, while $\boldsymbol{k}_{r}$ is the unit vector, pointing into the fluid, normal to the surface $\partial \Lambda_{r}$ bounding particle $r$. The surface integral is calculated over the dummy variable $z$.

The force $n\langle\boldsymbol{f}\rangle_{p}$ arises from the gradients of the point velocity of the ambient fluid present over the surface of the particles. Following Nott et al. [28], we divide this force into two parts: one related to the distortion of the fluid streamlines caused by the particles and one related to the lubrication films between particle pairs. Accordingly, we write:

$$
n\langle\boldsymbol{f}\rangle_{p}=n\langle\boldsymbol{f}\rangle_{p}^{\star}+n\langle\boldsymbol{f}\rangle_{p}^{\bullet}
$$

The second term on the right is the contribution of the lubrication films, which vanishes in dilute suspensions.

Now, in a dense suspension, let us consider particle $r$. We may assume this is located very close to a number of other particles with which it forms lubrication films. Each film spans a tiny portion of the particle surface, so for convenience we regard these portions as points. Since the fluid dynamic force acting on these small regions is finite, we must assume that in these points the stress diverges. In the rest of the particle surface, where lubrication films are absent, the stress is finite. But if lubrication regions are points, then the rest of the particle surface coincides with the entire particle surface (for a finite collection of points occupies no surface). Therefore, we can write:

$$
\begin{aligned}
& n(\boldsymbol{x}, t)\langle\boldsymbol{f}\rangle_{p}^{\star}(\boldsymbol{x}, t) \equiv-\sum_{r}\left[\psi\left(\left|\boldsymbol{x}-\boldsymbol{z}_{r}(t)\right|\right) \int_{\partial \Lambda_{r}} \boldsymbol{k}_{r}(z, t) \cdot \boldsymbol{\sigma}_{e}^{\star}(\boldsymbol{z}, t) d \boldsymbol{s}_{z}\right], \\
& n(\boldsymbol{x}, t)\langle\boldsymbol{f}\rangle_{p}^{\bullet}(\boldsymbol{x}, t) \equiv-\sum_{r}\left[\psi\left(\left|\boldsymbol{x}-\boldsymbol{z}_{r}(t)\right|\right) \int_{\partial \Lambda_{r}} \boldsymbol{k}_{r}(z, t) \cdot \boldsymbol{\sigma}_{e}^{\bullet}(z, t) d \boldsymbol{s}_{z}\right] .
\end{aligned}
$$


Over the particle surface, except for the points where lubrication films are located, $\sigma_{e}^{\star}=\sigma_{e}$; the value of $\sigma_{e}^{\star}$ in the points just mentioned is immaterial, since $\sigma_{e}^{\star}$ is not a delta function. On the other hand, over the particle surface $\sigma_{e}^{\bullet}$ is nonzero solely where lubrication films are located (and, in these point regions, it diverges).

Many constitutive equations are available for the part of $n\langle f\rangle_{p}$ related to the distortion of the streamlines. Here we report the equation below, accounting for the buoyancy, drag, lift, and virtual mass forces:

$n\langle\boldsymbol{f}\rangle_{p}^{\star}=-\varepsilon_{s} \partial_{\boldsymbol{x}} \cdot\langle\boldsymbol{S}\rangle_{e}+\beta\left(\langle\boldsymbol{u}\rangle_{e}-\langle\boldsymbol{u}\rangle_{s}\right)+\varepsilon_{s} \rho_{e} \zeta\left[\left(D_{t}\langle\boldsymbol{u}\rangle_{e}-D_{t}\langle\boldsymbol{u}\rangle_{s}\right)-\left(\partial_{\boldsymbol{x}} \mathbf{x}\langle\boldsymbol{u}\rangle_{e}\right) \mathbf{x}\left(\langle\boldsymbol{u}\rangle_{e}-\langle\boldsymbol{u}\rangle_{s}\right)\right]$.

The drag coefficient $\beta$ can be closed with Eq. (18), a relation suggested by Wen and Yu [43], but many other options are available in the literature. $\zeta$ is the coefficient for the virtual mass and lift forces, often assumed to be equal to $1 / 2$.

Equation (41), which defines the part of the fluid-particle interaction force related to lubrication films, can be written as:

$$
n(\boldsymbol{x}, t)\langle\boldsymbol{f}\rangle_{p}^{\bullet}(\boldsymbol{x}, t)=-\sum_{r}\left[\psi\left(\left|\boldsymbol{x}-\boldsymbol{z}_{r}(t)\right|\right) \sum_{s \neq r} \boldsymbol{f}_{r s}^{\bullet}(t)\right],
$$

where $f_{r s}^{\bullet}$ is the force exerted by particle $r$ on particle $s$ through the lubrication film between them; this force is equal and opposite to that exerted by particle $s$ on particle $r$ through the same lubrication film. The second summation is over all the particles $s$ with which particle $r$ forms lubrication films. Now, with $2 z_{r s} \equiv z_{s}-z_{r}$, the following relation holds:

$$
\sum_{r}\left[\psi\left(\left|\boldsymbol{x}-\boldsymbol{z}_{r s}(t)\right|\right) \sum_{s \neq r} \boldsymbol{f}_{r s}^{\bullet}(t)\right]=\mathbf{0} .
$$

We now expand the weighting function in a Taylor series around the center $z_{r}$ of the generic particle $r$. Since lubrication films are thin, $\boldsymbol{z}_{r s}-\boldsymbol{z}_{r} \approx a \boldsymbol{k}_{r s}$. Thus, it is:

$$
\begin{aligned}
\psi\left(\left|\boldsymbol{x}-\boldsymbol{z}_{r s}(t)\right|\right)= & \psi\left(\left|\boldsymbol{x}-\boldsymbol{z}_{r}(t)\right|\right)-a \boldsymbol{k}_{r s}(t) \cdot \partial_{\boldsymbol{x}} \psi\left(\left|\boldsymbol{x}-\boldsymbol{z}_{r}(t)\right|\right) \\
& +(1 / 2) a^{2} \boldsymbol{k}_{r s}(t) \boldsymbol{k}_{r s}(t): \partial_{\boldsymbol{x}} \partial_{\boldsymbol{x}} \psi\left(\left|\boldsymbol{x}-\boldsymbol{z}_{r}(t)\right|\right)-\cdots
\end{aligned}
$$

The particle radius $a$ is far smaller than the radius of the weighting function (which in turn is far smaller than the shortest significant macroscopic length scale $L$ associated with the flow); so, with small error, we can truncate the Taylor series at the second-order term. Then, if we introduce Eq. (45) in Eq. (44) and use Eq. (43), we obtain:

$$
n\langle\boldsymbol{f}\rangle_{p}^{\bullet}=-\partial_{x} \cdot\langle\boldsymbol{M}\rangle_{e}
$$

with:

$$
\langle\boldsymbol{M}\rangle_{e}(\boldsymbol{x}, t) \equiv n(\boldsymbol{x}, t)\langle\boldsymbol{E}\rangle_{p}(\boldsymbol{x}, t)-(1 / 2) \partial_{\boldsymbol{x}} \cdot\left[n(\boldsymbol{x}, t)\langle\boldsymbol{F}\rangle_{p}(\boldsymbol{x}, t)\right]
$$

and where:

$$
\begin{gathered}
n(\boldsymbol{x}, t)\langle\boldsymbol{E}\rangle_{p}(\boldsymbol{x}, t) \equiv a \sum_{r}\left[\psi\left(\left|\boldsymbol{x}-\boldsymbol{z}_{r}(t)\right|\right) \sum_{s \neq r} \boldsymbol{k}_{r s}(t) \boldsymbol{f}_{r s}^{\bullet}(t)\right], \\
n(\boldsymbol{x}, t)\langle\boldsymbol{F}\rangle_{p}(\boldsymbol{x}, t) \equiv a^{2} \sum_{r}\left[\psi\left(\left|\boldsymbol{x}-\boldsymbol{z}_{r}(t)\right|\right) \sum_{s \neq r} \boldsymbol{k}_{r s}(t) \boldsymbol{k}_{r s}(t) \boldsymbol{f}_{r s}^{\bullet}(t)\right] .
\end{gathered}
$$

Equation (39) thus becomes:

$$
n\langle\boldsymbol{f}\rangle_{p}=n\langle\boldsymbol{f}\rangle_{p}^{\star}-\partial_{\boldsymbol{x}} \cdot\langle\boldsymbol{M}\rangle_{e}
$$


The derivation of Eq. (17) neglects the second term on the right-hand side of Eq. (50). Details are found in Jamshidi et al. [14]. If this term is included, then one obtains:

$$
\langle\boldsymbol{u}\rangle_{s}-\langle\boldsymbol{u}\rangle_{e}=-(1 / \beta)\left\{\varepsilon_{e} \partial_{\boldsymbol{x}} \cdot\left[\langle\boldsymbol{S}\rangle_{s}+\langle\boldsymbol{M}\rangle_{e}\right]-\varepsilon_{s}\left(\rho_{s}-\rho_{m}\right)\left(\boldsymbol{g}-D_{t}\langle\boldsymbol{u}\rangle_{m}\right)\right\} .
$$

Based on what we discussed before, we see that in suspensions of neutrally buoyant particles changes in space of the tensor $\langle\boldsymbol{M}\rangle_{e}$ are the main cause for particle migration.

Following similar passages to those shown for the force $n\langle f\rangle_{p}$, we split the particle-presence stress tensor in two parts, writing:

$$
\begin{aligned}
& n(\boldsymbol{x}, t)\langle\boldsymbol{A}\rangle_{p}(\boldsymbol{x}, t)=n(\boldsymbol{x}, t)\langle\boldsymbol{A}\rangle_{p}^{\star}(\boldsymbol{x}, t)+n(\boldsymbol{x}, t)\langle\boldsymbol{A}\rangle_{p}^{\bullet}(\boldsymbol{x}, t), \\
& n(\boldsymbol{x}, t)\langle\boldsymbol{B}\rangle_{p}(\boldsymbol{x}, t)=n(\boldsymbol{x}, t)\langle\boldsymbol{B}\rangle_{p}^{\star}(\boldsymbol{x}, t)+n(\boldsymbol{x}, t)\langle\boldsymbol{B}\rangle_{p}^{\bullet}(\boldsymbol{x}, t) .
\end{aligned}
$$

The two starred terms involve $\boldsymbol{\sigma}_{e}^{\star}$ and are related to the viscous dissipation generated by the distortion of the fluid streamlines around the particles; these terms are always present, also in very dilute suspensions, where they give the Einstein correction for the mixture viscosity [34]. The other two terms account for the viscous dissipation associated with the lubrication films and are dominant in dense suspensions. From Eqs. (4) and (5), it is:

$$
\begin{gathered}
n(\boldsymbol{x}, t)\langle\boldsymbol{A}\rangle_{p}^{\bullet}(\boldsymbol{x}, t) \equiv a \sum_{r}\left[\psi\left(\left|\boldsymbol{x}-\boldsymbol{z}_{r}(t)\right|\right) \int_{\partial \Lambda_{r}} \boldsymbol{k}_{r}(\boldsymbol{z}, t) \boldsymbol{k}_{r}(z, t) \cdot \boldsymbol{\sigma}_{e}^{\bullet}(z, t) d \boldsymbol{s}_{z}\right], \\
n(\boldsymbol{x}, t)\langle\boldsymbol{B}\rangle_{p}^{\bullet}(\boldsymbol{x}, t) \equiv a^{2} \sum_{r}\left[\psi\left(\left|\boldsymbol{x}-\boldsymbol{z}_{r}(t)\right|\right) \int_{\partial \Lambda_{r}} \boldsymbol{k}_{r}(z, t) \boldsymbol{k}_{r}(z, t) \boldsymbol{k}_{r}(\boldsymbol{z}, t) \cdot \boldsymbol{\sigma}_{e}^{\bullet}(\boldsymbol{z}, t) d \boldsymbol{s}_{z}\right] .
\end{gathered}
$$

But being $\sigma_{e}^{\bullet}$ a delta function in a numerable set of points over the surfaces of the particles, these expressions reduce to those in Eqs. (48) and (49). So, it is:

$$
\langle\boldsymbol{M}\rangle_{e}(\boldsymbol{x}, t)=n(\boldsymbol{x}, t)\langle\boldsymbol{A}\rangle_{p}^{\bullet}(\boldsymbol{x}, t)-(1 / 2) \partial_{\boldsymbol{x}} \cdot\left[n(\boldsymbol{x}, t)\langle\boldsymbol{B}\rangle_{p}^{\bullet}(\boldsymbol{x}, t)\right],
$$

As anticipated, we see that $\langle\boldsymbol{M}\rangle_{e}$ coincides with the part of the particle-presence stress tensor related to the lubrication films. In suspensions of neutrally buoyant particles-in conditions where direct particle contacts and interparticle forces are negligible - the divergence of $\langle\boldsymbol{M}\rangle_{e}$ is the main driver of migration.

\section{A. More on the closure of Morris and Boulay}

Morris and Boulay [15] suggested their closure for the effective stress tensor of the solid on the assumptions that (a) shear-induced particle migration is caused by nonuniformity in this tensor and (b) the dominant stresses in the solid phase originate from the lubrication forces between the particles. Our analysis suggests that their constitutive equation should be used to close the tensor $\langle\boldsymbol{M}\rangle_{e}$, which is part of the fluid effective stress tensor $\langle\boldsymbol{S}\rangle_{e}$.

Let us consider again the steady wide-gap Couette flow. Equation (31) still holds. If for the solid phase we neglect the kinetic stress induced by particle velocity fluctuations, the solid effective stress tensor vanishes and Eq. (31b) is automatically satisfied. Thus, we need a closure only for $\langle\boldsymbol{S}\rangle_{e}$. This tensor is defined by Eq. (3), shown here for convenience:

$$
\langle\boldsymbol{S}\rangle_{e} \equiv \varepsilon_{e}\langle\boldsymbol{\sigma}\rangle_{e}+\varepsilon_{e} \rho_{e}\langle\hat{\boldsymbol{u}} \hat{\boldsymbol{u}}\rangle_{e}+n\langle\boldsymbol{A}\rangle_{p}-(1 / 2) \partial_{\boldsymbol{x}} \cdot\left(n\langle\boldsymbol{B}\rangle_{p}\right)
$$

For the flow investigated, the Reynolds stress is negligible. Also, as discussed in Jackson [34], the first term on the right is closed and equal to:

$$
\varepsilon_{e}\langle\boldsymbol{\sigma}\rangle_{e}=\varepsilon_{e}\langle p\rangle_{e} \boldsymbol{I}-\mu_{e}\langle\dot{\boldsymbol{\gamma}}\rangle_{v}
$$

in which $\langle p\rangle_{e}$ is the fluid mean pressure, $\boldsymbol{I}$ is the unit tensor, and $\langle\dot{\gamma}\rangle_{v}$ is twice the deformation rate tensor of the following velocity field:

$$
\langle\boldsymbol{u}\rangle_{v} \equiv \varepsilon_{e}\langle\boldsymbol{u}\rangle_{e}+\varepsilon_{s}\langle\boldsymbol{u}\rangle_{s}
$$


For mixtures of neutrally buoyant particles (where the velocity fields relax rapidly), the mean velocities of the phases are equal at leading order in the Stokes number and so $\langle\boldsymbol{u}\rangle_{v}$ and $\langle\boldsymbol{u}\rangle_{m}$ coincide. The last two terms on the right-hand side of Eq. (3) embody the particle-presence stress tensor. If we neglect the viscous part related to the distortion of the fluid streamlines, an assumption that can be accepted in dense mixtures, then we obtain:

$$
n\langle\boldsymbol{A}\rangle_{p}-(1 / 2) \partial_{\boldsymbol{x}} \cdot\left(n\langle\boldsymbol{B}\rangle_{p}\right) \approx \varepsilon_{s}\langle p\rangle_{e} \boldsymbol{I}+\langle\boldsymbol{M}\rangle_{e} .
$$

Employing the constitutive equation of Morris and Boulay [15] to express the tensor $\langle\boldsymbol{M}\rangle_{e}$, we then obtain:

$$
\langle\boldsymbol{S}\rangle_{e}=\langle p\rangle_{e} \boldsymbol{I}-\eta_{s}\left(\varepsilon_{s}\right)\langle\dot{\boldsymbol{\gamma}}\rangle_{m}+\eta_{n}\left(\varepsilon_{s}\right)\left|\langle\dot{\boldsymbol{\gamma}}\rangle_{m}\right| \boldsymbol{Q},
$$

where, as mentioned before, $\eta_{s}\left(\varepsilon_{s}\right) \equiv \mu_{e}+\eta_{p}\left(\varepsilon_{s}\right)$ denotes the shear viscosity of the mixture. Reminding that the dynamical equation for the solid phase is automatically satisfied, the only equation which has to be solved to determine the steady-state solid volume fraction profile in the wide-gap Couette flow is:

$$
\partial_{\boldsymbol{x}} \cdot\left[\eta_{s}\left(\varepsilon_{s}\right)\langle\dot{\boldsymbol{\gamma}}\rangle_{m}-\eta_{n}\left(\varepsilon_{s}\right)\left|\langle\dot{\boldsymbol{\gamma}}\rangle_{m}\right| \boldsymbol{Q}\right]=\partial_{\boldsymbol{x}}\langle p\rangle_{e}-\rho_{m} \boldsymbol{g} .
$$

This equation differs from Eq. (35), but only because of the gravitational and pressure terms on the right-hand side of the equation above. These affect the vertical pressure distribution, but vanish in the $\theta$ direction. Hence, they do not alter the solid volume fraction profile, given by Eq. (31a). We thus recover the results obtained by Morris and Boulay [15].

\section{B. The closure of Gillissen and Wilson}

Another constitutive equation that can be employed for the tensor $\langle\boldsymbol{M}\rangle_{e}$ has been recently derived by Gillissen and Wilson [47], who developed a model for the microstructure and stress in dense suspensions of non-Brownian, smooth spheres at vanishing small particle Reynolds number. Their closure reads:

$$
\langle\boldsymbol{M}\rangle_{e}=-\alpha\left(\varepsilon_{s}\right) \mu_{e}\langle\dot{\gamma}\rangle_{m}:\langle\boldsymbol{p p p p}\rangle,
$$

where $\alpha\left(\varepsilon_{s}\right)$, referred to as stress magnitude, is a function that diverges as the mixture nears maximum packing, $\boldsymbol{p}$ is the orientation unit vector, which joins the centers of particle pairs separated by lubrication films, while $\langle\cdots\rangle$ is the average operator involving the probability density function of the vector $\boldsymbol{p}$ between interacting particles. To calculate the fourth-order moment $\langle\boldsymbol{p p p p}\rangle$, one must first evaluate the second-order moment $\langle\boldsymbol{p} \boldsymbol{p}\rangle$, which is referred to as microstructure tensor. An evolution equation for the latter is reported in Ref. [47], to which we refer for further details. Equation (62) successfully captures the typical non-Newtonian behavior of dense mixtures, including normal stress differences in steady shear flows and time-dependent stress in abruptly reversed shear flows and oscillating shear flows.

\section{CONCLUSIONS}

In its original form, the mixture model suggests that shear-induced migration of neutrally buoyant particles is induced solely by gradients in the solid effective stress tensor. In dense mixtures where direct particle contacts and interparticle forces are negligible, the solid stress arises from the particle velocity fluctuations generated when adjacent suspension layers move past one another, and thus coincides with the solid kinetic stress. Using Buyevich's [3] closure for the solid kinetic stress tensor, we found that the time needed by the kinetic stress to cause appreciable particle migration exceeds significantly the timescale of the migration process, a result showing that, if direct particle contacts and interparticle forces are negligible, particle migration must have another driver. Many believe that this is the lubrication forces between the particles. On this assumption, Morris and Boulay [15] proposed a constitutive equation for the solid effective stress tensor, a closure that 
seems to predict well the migration process. However, their equation poses a conceptual problem, because the effect of lubrication forces is captured by the effective stress tensor of the fluid, not of the solid, a matter thoroughly discussed by Nott et al. [28]. Building on their ideas, we investigated this aspect further, showing that lubrication forces may induce particle migration, and the tensor quantifying their effect (the part of the particle-presence stress tensor related to lubrication films) can be closed by Morris and Boulay's constitutive equation.

\section{ACKNOWLEDGMENTS}

The authors acknowledge the EPSRC (Grant No. EP/N024915/1) for the financial support offered for this work.

\section{APPENDIX A: DERIVATION OF THE TWO-FLUID EQUATIONS OF MOTION}

In this Appendix, we derive the two-fluid equations of motion using volume averages; the treatment is similar to that of Jackson [34], but for the solid phase, instead of using particle-phase averages, we adopt solid-phase averages, so that the fluid and solid phases are treated equally. This choice allows connecting the results of this analysis to those of Batchelor [36] for particle suspensions. The results are equivalent to those derived by Zhang and Prosperetti [35] via ensemble averaging.

\section{Fluid phase}

The fluid volume fraction and the fluid-phase volume average of a generic point variable $\zeta(x, t)$ are defined as follows:

$$
\varepsilon_{e}(\boldsymbol{x}, t) \equiv \int_{\Lambda_{e}} \psi(|\boldsymbol{x}-z|) d z ; \quad\langle\zeta\rangle_{e}(\boldsymbol{x}, t) \equiv \frac{1}{\varepsilon_{e}(\boldsymbol{x}, t)} \int_{\Lambda_{e}} \zeta(z, t) \psi(|\boldsymbol{x}-z|) d z
$$

where $\Lambda_{e}$ is the domain occupied by the fluid phase at time $t$ and $\psi(|x-z|)$ is the weighting function. Now, consider the equation of motion for the fluid:

$$
\rho_{e} \partial_{t} \boldsymbol{u}_{e}=-\rho_{e} \partial_{\boldsymbol{x}} \cdot\left(\boldsymbol{u}_{e} \boldsymbol{u}_{e}\right)-\partial_{\boldsymbol{x}} \cdot \boldsymbol{\sigma}_{e}+\rho_{e} \boldsymbol{g}
$$

where we have assumed the fluid is incompressible. Here $\boldsymbol{u}_{e}$ and $\boldsymbol{\sigma}_{e}$ are the point (that is, nonaveraged) velocity and stress tensor of the fluid. Let us multiply both sides by $\psi(|x-z|)$ and integrate over $\Lambda_{e}$ with respect to $z$; this gives:

$$
\varepsilon_{e} \rho_{e}\left\langle\partial_{t} \boldsymbol{u}\right\rangle_{e}=-\varepsilon_{e} \rho_{e}\left\langle\partial_{\boldsymbol{x}} \cdot(\boldsymbol{u} \boldsymbol{u})\right\rangle_{e}-\varepsilon_{e}\left\langle\partial_{\boldsymbol{x}} \cdot \boldsymbol{\sigma}\right\rangle_{e}+\varepsilon_{e} \rho_{e} \boldsymbol{g} .
$$

To manipulate it, we use the following transport theorems, whose proofs are in the literature (for instance, see Ref. [48] or Ref. [49]):

$$
\begin{gathered}
\varepsilon_{e}\left\langle\partial_{t} \boldsymbol{u}\right\rangle_{e}+\varepsilon_{e}\left\langle\partial_{\boldsymbol{x}} \cdot(\boldsymbol{u} \boldsymbol{u})\right\rangle_{e}=\partial_{t}\left(\varepsilon_{e}\langle\boldsymbol{u}\rangle_{e}\right)+\partial_{\boldsymbol{x}} \cdot\left(\varepsilon_{e}\langle\boldsymbol{u} \boldsymbol{u}\rangle_{e}\right), \\
\varepsilon_{e}\left\langle\partial_{\boldsymbol{x}} \cdot \boldsymbol{\sigma}\right\rangle_{e}=\partial_{\boldsymbol{x}} \cdot\left(\varepsilon_{e}\langle\boldsymbol{\sigma}\rangle_{e}\right)-\sum_{r} \int_{\partial \Lambda_{r}} \boldsymbol{k}_{r}(z, t) \cdot \boldsymbol{\sigma}_{e}(z, t) \psi(|\boldsymbol{x}-z|) d \boldsymbol{s}_{z} .
\end{gathered}
$$

With these results, we can write Eq. (A3) as follows:

$$
\rho_{e} \partial_{t}\left(\varepsilon_{e}\langle\boldsymbol{u}\rangle_{e}\right)=-\rho_{e} \partial_{\boldsymbol{x}} \cdot\left(\varepsilon_{e}\langle\boldsymbol{u} \boldsymbol{u}\rangle_{e}\right)-\partial_{\boldsymbol{x}} \cdot\left(\varepsilon_{e}\langle\boldsymbol{\sigma}\rangle_{e}\right)+\sum_{r} \int_{\partial \Lambda_{r}} \boldsymbol{k}_{r} \cdot \boldsymbol{\sigma}_{e} \psi(|\boldsymbol{x}-\boldsymbol{z}|) d \boldsymbol{s}_{z}+\varepsilon_{e} \rho_{e} \boldsymbol{g}
$$

We now arrive at a key point of the analysis: The third term on the right-hand side does not represent the mean fluid-particle interaction force. The definition that reflects the physical meaning we ascribe to the mean force exerted by the fluid on the particles and that is used in numerical simulations to 
calculate this force (for instance, see Ref. [50] and Ref. [51]) is as follows:

$$
n(\boldsymbol{x}, t)\langle\boldsymbol{f}\rangle_{p}(\boldsymbol{x}, t) \equiv-\sum_{r}\left[\int_{\partial \Lambda_{r}} \boldsymbol{k}_{r}(z, t) \cdot \boldsymbol{\sigma}_{e}(z, t) d \boldsymbol{s}_{z}\right] \psi\left(\left|\boldsymbol{x}-\boldsymbol{z}_{r}(t)\right|\right),
$$

where $z_{r}$ is the location of the center of particle $r$ at time $t$. In Eq. (A7), one first calculates the force exerted by the fluid on particle $r$, after weights it using the value of the weighting function in $z_{r}$, and finally sums over all the particles in the suspension. By contrast, in the corresponding term in Eq. (A6), the fluid-particle interaction force on each differential surface element of each particle is weighted using the value of the weighting function at the location where the infinitesimal force acts. To make $n\langle\boldsymbol{f}\rangle_{p}$ appear in Eq. (A6), we expand $\psi$ in a Taylor series about $\boldsymbol{z}_{r}$, obtaining:

$$
\sum_{r} \int_{\partial \Lambda_{r}} \boldsymbol{k}_{r} \cdot \boldsymbol{\sigma}_{e} \psi(|\boldsymbol{x}-\boldsymbol{z}|) d \boldsymbol{s}_{z}=-n\langle\boldsymbol{f}\rangle_{p}-\partial_{\boldsymbol{x}} \cdot\left[n\langle\boldsymbol{A}\rangle_{p}-(1 / 2) \partial_{\boldsymbol{x}} \cdot\left(n\langle\boldsymbol{B}\rangle_{p}\right)\right],
$$

where $n\langle\boldsymbol{A}\rangle_{p}$ and $n\langle\boldsymbol{B}\rangle_{p}$ are defined by Eqs. (4) and (5). The tensor appearing in square brackets in Eq. (A8) is referred to as particle-presence stress and is related to the direct fluid-particle interaction occurring on the surfaces of the particles. Using Eq. (A8), we obtain:

$$
\begin{aligned}
\rho_{e} \partial_{t}\left(\varepsilon_{e}\langle\boldsymbol{u}\rangle_{e}\right)= & -\rho_{e} \partial_{\boldsymbol{x}} \cdot\left(\varepsilon_{e}\langle\boldsymbol{u}\rangle_{e}\langle\boldsymbol{u}\rangle_{e}\right)-\partial_{\boldsymbol{x}} \cdot\left[\varepsilon_{e}\langle\boldsymbol{\sigma}\rangle_{e}+\varepsilon_{e} \rho_{e}\langle\hat{\boldsymbol{u}} \hat{\boldsymbol{u}}\rangle_{e}+n\langle\boldsymbol{A}\rangle_{p}\right. \\
& \left.-(1 / 2) \partial_{\boldsymbol{x}} \cdot\left(n\langle\boldsymbol{B}\rangle_{p}\right)\right]-n\langle\boldsymbol{f}\rangle_{p}+\varepsilon_{e} \rho_{e} \boldsymbol{g},
\end{aligned}
$$

where we have decomposed the convective term into the sum of two terms involving the dyad of the average velocities and the average of the velocity fluctuations dyad. In this form, the equation reveals that the expression of the fluid effective stress tensor is given by Eq. (3). For liquid-particle suspensions, usually the dominant term is $n\langle\boldsymbol{A}\rangle_{p}$, the leading part of the particle-presence stress tensor.

\section{Solid phase}

For the dispersed phase, one can adopt two types of averages. The solid volume fraction and the solid-phase volume average of a generic point variable $\zeta(\boldsymbol{x}, t)$ are defined as follows:

$$
\varepsilon_{s}(\boldsymbol{x}, t) \equiv \sum_{r} \int_{\Lambda_{r}} \psi(|\boldsymbol{x}-\boldsymbol{z}|) d \boldsymbol{z} ; \quad\langle\zeta\rangle_{s}(\boldsymbol{x}, t) \equiv \frac{1}{\varepsilon_{s}(\boldsymbol{x}, t)} \sum_{r} \int_{\Lambda_{r}} \zeta(z, t) \psi(|\boldsymbol{x}-\boldsymbol{z}|) d z .
$$

The particle number density and the particle-phase volume average of a generic property $\zeta_{r}(t)$ of (the entire) particle $r$ are defined as follows:

$$
n(\boldsymbol{x}, t) \equiv \sum_{r} \psi\left(\left|\boldsymbol{x}-\boldsymbol{z}_{r}(t)\right|\right) ; \quad\langle\zeta\rangle_{p}(\boldsymbol{x}, t) \equiv \frac{1}{n(\boldsymbol{x}, t)} \sum_{r} \zeta_{r}(t) \psi\left(\left|\boldsymbol{x}-\boldsymbol{z}_{r}(t)\right|\right) .
$$

These averages are discussed in detail in the literature [35]. Here, we use solid-phase averages, so that the fluid and solid phases are treated equally. Consider the equation of motion for the solid phase, which holds in every point occupied by the solid material:

$$
\rho_{s} \partial_{t} \boldsymbol{u}_{s}=-\rho_{s} \partial_{\boldsymbol{x}} \cdot\left(\boldsymbol{u}_{s} \boldsymbol{u}_{s}\right)-\partial_{\boldsymbol{x}} \cdot \boldsymbol{\sigma}_{s}+\rho_{s} \boldsymbol{g}
$$

Here $\boldsymbol{u}_{s}$ and $\boldsymbol{\sigma}_{s}$ denote the point velocity and stress tensor of the solid. Multiply both sides by $\psi(|x-z|)$ and integrate with respect to $z$ over the region of space occupied by the particles; this gives:

$$
\rho_{s} \varepsilon_{s}\left\langle\partial_{t} \boldsymbol{u}\right\rangle_{s}=-\rho_{s} \varepsilon_{s}\left\langle\partial_{\boldsymbol{x}} \cdot(\boldsymbol{u} \boldsymbol{u})\right\rangle_{s}-\varepsilon_{s}\left\langle\partial_{\boldsymbol{x}} \cdot \boldsymbol{\sigma}\right\rangle_{s}+\varepsilon_{s} \rho_{s} \boldsymbol{g}
$$


An analogous version of Eq. (A4) holds; this allows us to manipulate the accumulation and convective terms. Also, using Eq. (A10), we can write:

$$
\varepsilon_{s}\left\langle\partial_{x} \cdot \sigma\right\rangle_{s} \equiv \sum_{r} \int_{\Lambda_{r}} \partial_{z} \cdot \sigma_{s}(z, t) \psi(|x-z|) d z
$$

Now we expand the weighting function in a Taylor series about the center $z_{r}$ of the generic particle $r$. Doing so yields the following series, of which we retain the first two terms:

$$
\begin{aligned}
\varepsilon_{s}\left\langle\partial_{\boldsymbol{x}} \cdot \boldsymbol{\sigma}\right\rangle_{s}= & \sum_{r}\left[\int_{\Lambda_{r}} \partial_{z} \cdot \boldsymbol{\sigma}_{s} d z\right] \psi\left(\left|\boldsymbol{x}-\boldsymbol{z}_{r}\right|\right)-\partial_{\boldsymbol{x}} \cdot \sum_{r}\left[\int_{\Lambda_{r}}\left(z-z_{r}\right) \partial_{z} \cdot \boldsymbol{\sigma}_{s} d z\right] \psi\left(\left|\boldsymbol{x}-\boldsymbol{z}_{r}\right|\right) \\
& +(1 / 2) \partial_{\boldsymbol{x}} \partial_{\boldsymbol{x}}: \sum_{r}\left[\int_{\Lambda_{r}}\left(z-z_{r}\right)\left(z-z_{r}\right) \partial_{z} \cdot \sigma_{s} d z\right] \psi\left(\left|\boldsymbol{x}-\boldsymbol{z}_{r}\right|\right)-\cdots
\end{aligned}
$$

In the first term on the right-hand side, we may manipulate the volume integral using the divergence theorem. Doing so yields:

$$
\int_{\Lambda_{r}} \partial_{z} \cdot \sigma_{s} d z=\int_{\partial \Lambda_{r}} \boldsymbol{k}_{r} \cdot \boldsymbol{\sigma}_{s} d \boldsymbol{s}_{z}
$$

Notice that the surface integral on the right-hand side of this equation is not equal to the force that the particle exerts on the surrounding fluid, insofar as the stress tensor featuring in the integrand is that pertaining to the solid material. Because the particle exerts a force also on the particles with which it is in direct contact at any given time, the surface integral above differs from the fluid-particle interaction force. To eliminate the stress tensor of the solid material, and make the fluid-particle interaction force appear, we use the linear momentum jump condition holding at the particle surface:

$$
\boldsymbol{k}_{r} \cdot \boldsymbol{\sigma}_{s}=\boldsymbol{k}_{r} \cdot \boldsymbol{\sigma}_{e}+\boldsymbol{c}_{r} .
$$

Here $c_{r}$ denotes the surface density of the contact force that particle $r$ exerts on the particles that are in direct contact with it. Then, we obtain:

$$
\sum_{r}\left[\int_{\Lambda_{r}} \partial_{z} \cdot \sigma_{s} d z\right] \psi\left(\left|\boldsymbol{x}-\boldsymbol{z}_{r}\right|\right)=\sum_{r}\left[\int_{\partial \Lambda_{r}} \boldsymbol{k}_{r} \cdot \boldsymbol{\sigma}_{e} d \boldsymbol{s}_{z}+\int_{\partial \Lambda_{r}} \boldsymbol{c}_{r} d \boldsymbol{s}_{z}\right] \psi\left(\left|\boldsymbol{x}-\boldsymbol{z}_{r}\right|\right) .
$$

The term associated with the first integral on the right-hand side is the average force that the particles exert on the fluid. For the other term, it is:

$$
\sum_{r}\left[\int_{\partial \Lambda_{r}} \boldsymbol{c}_{r} d \boldsymbol{s}_{z}\right] \psi\left(\left|\boldsymbol{x}-\boldsymbol{z}_{r}\right|\right)=\sum_{r}\left[\sum_{s \neq r} \boldsymbol{f}_{r s}\right] \psi\left(\left|\boldsymbol{x}-\boldsymbol{z}_{r}\right|\right)=\partial_{\boldsymbol{x}} \cdot\left[n\langle\boldsymbol{C}\rangle_{p}-(1 / 2) \partial_{\boldsymbol{x}} \cdot\left(n\langle\boldsymbol{D}\rangle_{p}\right)\right] .
$$

The last passage is proved in Mazzei [52]. Here $\boldsymbol{f}_{r s}$ is the direct contact force that particle $r$ exerts on particle $s$. Furthermore, the tensors $n\langle\boldsymbol{C}\rangle_{p}$ and $n\langle\boldsymbol{D}\rangle_{p}$ are defined by Eqs. (9) and (10). With these results, we finally obtain:

$$
\sum_{r}\left[\int_{\Lambda_{r}} \partial_{z} \cdot \boldsymbol{\sigma}_{s} d \boldsymbol{z}\right] \psi\left(\left|\boldsymbol{x}-\boldsymbol{z}_{r}\right|\right)=-n\langle\boldsymbol{f}\rangle_{p}+\partial_{\boldsymbol{x}} \cdot\left[n\langle\boldsymbol{C}\rangle_{p}-(1 / 2) \partial_{\boldsymbol{x}} \cdot\left(n\langle\boldsymbol{D}\rangle_{p}\right)\right] .
$$

To eliminate the stress tensor of the solid material from the second term featuring on the right-hand side of Eq. (A15), we use Eq. (A12), writing:

$$
\begin{aligned}
\sum_{r}\left[\int_{\Lambda_{r}}\left(z-z_{r}\right) \partial_{z} \cdot \sigma_{s} d z\right] \psi\left(\left|\boldsymbol{x}-\boldsymbol{z}_{r}\right|\right) & =\sum_{r}\left[\int_{\Lambda_{r}}\left(z-z_{r}\right)\left(\rho_{s} \boldsymbol{g}-\rho_{s} \dot{\boldsymbol{u}}_{s}\right) d \boldsymbol{z}\right] \psi\left(\left|\boldsymbol{x}-\boldsymbol{z}_{r}\right|\right) \\
& =-\sum_{r}\left[\int_{\Lambda_{r}}\left(z-\boldsymbol{z}_{r}\right)\left(\rho_{s} \dot{\boldsymbol{u}}_{s}\right) d z\right] \psi\left(\left|\boldsymbol{x}-\boldsymbol{z}_{r}\right|\right),
\end{aligned}
$$


in which $\dot{\boldsymbol{u}}_{s}$ denotes the substantial derivative of the local velocity of the particle material. The gravitational field gives no contribution because it is constant (and the particle is spherical). Since the particle is assumed to be rigid, the following kinematic equation holds:

$$
\dot{u}_{s}=\dot{u}_{r}-\left(z-z_{r}\right) \times \dot{\omega}_{r}-\left(z-z_{r}\right)\left(\omega_{r} \cdot \omega_{r}\right)+\left(z-z_{r}\right) \cdot \omega_{r} \omega_{r}
$$

where $\boldsymbol{u}_{r}$ is the velocity of the center of particle $r$ and $\omega_{r}$ is the angular velocity of particle $r$ (both functions only of $t$, differently from $\boldsymbol{u}_{s}$, which depends also on the space coordinates), while $\dot{\boldsymbol{u}}_{r}$ and $\dot{\omega}_{r}$ are their time derivatives. Replacing this in Eq. (A21) gives:

$$
\begin{aligned}
& \sum_{r}\left[\int_{\Lambda_{r}}\left(z-z_{r}\right) \partial_{z} \cdot \sigma_{s} d z\right] \psi\left(\left|\boldsymbol{x}-z_{r}\right|\right) \\
& \quad=(1 / 2) \sum_{r} \boldsymbol{I} \times I_{s} \dot{\boldsymbol{\omega}}_{r} \psi\left(\left|\boldsymbol{x}-\boldsymbol{z}_{r}\right|\right)+\left(I_{s} / 2\right)\left(n\langle\boldsymbol{\omega} \cdot \boldsymbol{\omega}\rangle_{p} \boldsymbol{I}-n\langle\boldsymbol{\omega} \boldsymbol{\omega}\rangle_{p}\right),
\end{aligned}
$$

where $\boldsymbol{I}$ is the unit tensor and $I_{s}$ is the moment of inertia of the particle. To manipulate the term involving the angular acceleration of the particle, we employ the angular momentum balance equation for the particle. Hence, we write:

$$
\begin{aligned}
\sum_{r} \boldsymbol{I} \times I_{s} \dot{\boldsymbol{\omega}}_{r} \psi\left(\left|\boldsymbol{x}-\boldsymbol{z}_{r}\right|\right)= & -\sum_{r}\left[\int_{\partial \Lambda_{r}} a \boldsymbol{I} \times \boldsymbol{k}_{r} \times\left(\boldsymbol{k}_{r} \cdot \boldsymbol{\sigma}_{e}\right) d \boldsymbol{s}_{z}\right] \psi\left(\left|\boldsymbol{x}-\boldsymbol{z}_{r}\right|\right) \\
& -\sum_{r}\left[\sum_{s \neq r} a \boldsymbol{I} \times \boldsymbol{k}_{r s} \times \boldsymbol{f}_{r s}\right] \psi\left(\left|\boldsymbol{x}-\boldsymbol{z}_{r}\right|\right) .
\end{aligned}
$$

Since it is:

$$
I \times a \times b=b a-a b
$$

for any vectors $\boldsymbol{a}$ and $\boldsymbol{b}$, if we use this tensorial relation and the definitions given in Eqs. (4) and (9), we reach the following result:

$$
\sum_{r} \boldsymbol{I} \times I_{s} \dot{\boldsymbol{\omega}}_{r} \psi\left(\left|\boldsymbol{x}-\boldsymbol{z}_{r}\right|\right)=\left(n\langle\boldsymbol{A}\rangle_{p}-n\langle\boldsymbol{A}\rangle_{p}^{T}\right)+\left(n\langle\boldsymbol{C}\rangle_{p}-n\langle\boldsymbol{C}\rangle_{p}^{T}\right)
$$

whence:

$$
\begin{aligned}
& \sum_{r}\left[\int_{\Lambda_{r}}\left(z-z_{r}\right) \partial_{z} \cdot \sigma_{s} d z\right] \psi\left(\left|\boldsymbol{x}-\boldsymbol{z}_{r}\right|\right) \\
& \quad=(1 / 2)\left[\left(n\langle\boldsymbol{A}\rangle_{p}-n\langle\boldsymbol{A}\rangle_{p}^{T}\right)+\left(n\langle\boldsymbol{C}\rangle_{p}-n\langle\boldsymbol{C}\rangle_{p}^{T}\right)+I_{s}\left(n\langle\boldsymbol{\omega} \cdot \boldsymbol{\omega}\rangle_{p} \boldsymbol{I}-n\langle\boldsymbol{\omega} \boldsymbol{\omega}\rangle_{p}\right)\right] .
\end{aligned}
$$

For convenience, we denote this tensor as $n\langle\boldsymbol{\Omega}\rangle_{p}$. We neglect the third term in Eq. (A15), since following Jackson [34], we omit terms that are small of $O\left(a^{2} / L^{2}\right)$ relative to those retained (where $L$ is the shortest significant macroscopic length scale associated with the flow). Thus, Eq. (A15) reduces to:

$$
\varepsilon_{s}\left\langle\partial_{\boldsymbol{x}} \cdot \boldsymbol{\sigma}\right\rangle_{s}=-n\langle\boldsymbol{f}\rangle_{p}+\partial_{\boldsymbol{x}} \cdot\left[n\langle\boldsymbol{C}\rangle_{p}-(1 / 2) \partial_{\boldsymbol{x}} \cdot\left(n\langle\boldsymbol{D}\rangle_{p}\right)-n\langle\boldsymbol{\Omega}\rangle_{p}\right] .
$$

Introducing all these results in Eq. (A13), and decomposing the convective term as we did for the fluid phase, leads to the following equation:

$$
\begin{aligned}
\rho_{s} \partial_{t}\left(\varepsilon_{s}\langle\boldsymbol{u}\rangle_{s}\right)= & -\rho_{s} \partial_{\boldsymbol{x}} \cdot\left(\varepsilon_{s}\langle\boldsymbol{u}\rangle_{s}\langle\boldsymbol{u}\rangle_{s}\right)-\partial_{\boldsymbol{x}} \cdot\left[\varepsilon_{s} \rho_{s}\langle\hat{\boldsymbol{u}} \hat{\boldsymbol{u}}\rangle_{s}+n\langle\boldsymbol{C}\rangle_{p}-(1 / 2) \partial_{\boldsymbol{x}} \cdot\left(n\langle\boldsymbol{D}\rangle_{p}\right)\right. \\
& \left.-n\langle\boldsymbol{\Omega}\rangle_{p}\right]+n\langle\boldsymbol{f}\rangle_{p}+\varepsilon_{s} \rho_{s} \boldsymbol{g} .
\end{aligned}
$$


The order of magnitude of $n\langle\boldsymbol{\Omega}\rangle_{p}$ depends on that of the particle mean angular velocity, $\langle\boldsymbol{\omega}\rangle_{p}$. If this and $\partial_{x} \times\langle\boldsymbol{u}\rangle_{e}$ have equal order of magnitude, a common situation for liquid-particle mixtures, $\left|\langle\boldsymbol{\omega}\rangle_{p}\right| /\left|\langle\boldsymbol{u}\rangle_{e}\right|$ is $O(1 / L)$ and $n\langle\boldsymbol{\Omega}\rangle_{p}$ is $O\left(a^{2} / L^{2}\right)$. So, this tensor can be neglected. Then, from Eq. (A29), we arrive at the definition of solid effective stress tensor given in Eq. (8).

Note that if to derive the equation of motion for the solid phase one uses particle-phase averages (for this, see Ref. [34]), the resulting expression for the solid effective stress tensor is as follows:

$$
\langle\boldsymbol{S}\rangle_{p}(\boldsymbol{x}, t) \equiv \varepsilon_{s}(\boldsymbol{x}, t) \rho_{s}\langle\hat{\boldsymbol{u}} \hat{\boldsymbol{u}}\rangle_{p}(\boldsymbol{x}, t)+n(\boldsymbol{x}, t)\langle\boldsymbol{C}\rangle_{p}(\boldsymbol{x}, t)-(1 / 2) \partial_{\boldsymbol{x}} \cdot\left[n(\boldsymbol{x}, t)\langle\boldsymbol{D}\rangle_{p}(\boldsymbol{x}, t)\right]
$$

So, when $\left|\langle\boldsymbol{\omega}\rangle_{p}\right| /\left|\langle\boldsymbol{u}\rangle_{e}\right|$ is $O(1 / L)$, we see that $\langle\boldsymbol{S}\rangle_{s}=\langle\boldsymbol{S}\rangle_{p}+O\left(a^{2} / L^{2}\right)$; thus, at the level of approximation adopted, the two tensors are equal.

\section{APPENDIX B: CONNECTION WITH THE WORK OF BATCHELOR}

In Batchelor [36], the goal is deriving an expression for the stress tensor of a fluid-particle suspension. The result [Eq. (4.5)] can be written as follows:

$$
\boldsymbol{\Sigma}=\varepsilon_{e}\langle p\rangle_{e} \boldsymbol{I}-\mu_{e}\langle\dot{\boldsymbol{\gamma}}\rangle_{v}+\boldsymbol{\Sigma}^{(p)}=\varepsilon_{e}\langle\boldsymbol{\sigma}\rangle_{e}+\boldsymbol{\Sigma}^{(p)} .
$$

As we can see, $\boldsymbol{\Sigma}^{(p)}$ groups together all the contributions to the effective stress tensor of the suspension related to the particles (plus the fluid-phase kinetic stress). $\boldsymbol{\Sigma}^{(p)}$ is referred to as particle stress tensor and comprises four terms:

$$
\boldsymbol{\Sigma}^{(p)}=\boldsymbol{\Sigma}_{1}^{(p)}+\boldsymbol{\Sigma}_{2}^{(p)}+\boldsymbol{\Sigma}_{3}^{(p)}+\boldsymbol{\Sigma}_{4}^{(p)},
$$

where:

$$
\begin{gathered}
\boldsymbol{\Sigma}_{1}^{(p)}(\boldsymbol{x}, t) \equiv(1 / V) a \sum_{r} \int_{\partial \Lambda_{r}} \boldsymbol{k}_{r}(\boldsymbol{z}, t) \boldsymbol{k}_{r}(z, t) \cdot \boldsymbol{\sigma}_{e}(\boldsymbol{z}, t) d \boldsymbol{s}_{z}, \\
\boldsymbol{\Sigma}_{2}^{(p)}(\boldsymbol{x}, t) \equiv(1 / V) \sum_{r} \int_{\partial \Lambda_{r}} \mu_{e}\left[\boldsymbol{u}_{e}(\boldsymbol{z}, t) \boldsymbol{k}_{r}(z, t)+\boldsymbol{k}_{r}(\boldsymbol{z}, t) \boldsymbol{u}_{e}(z, t)\right] d \boldsymbol{s}_{z}, \\
\boldsymbol{\Sigma}_{3}^{(p)}(\boldsymbol{x}, t) \equiv(1 / V) \sum_{r} \int_{\Lambda_{r}}\left(z-z_{r}\right) \rho_{s} \boldsymbol{a}_{s}(z, t) d z, \\
\boldsymbol{\Sigma}_{4}^{(p)}(\boldsymbol{x}, t) \equiv(1 / V) \int_{\Lambda_{e}} \rho_{e} \boldsymbol{u}_{e}(\boldsymbol{z}, t) \boldsymbol{u}_{e}(\boldsymbol{z}, t) d z+(1 / V) \sum_{r} \int_{\Lambda_{r}} \rho_{s} \boldsymbol{u}_{s}(\boldsymbol{z}, t) \boldsymbol{u}_{s}(z, t) d \boldsymbol{z} .
\end{gathered}
$$

Here $V$ denotes the volume of the region over which the average is performed (in Batchelor's analysis, this is the entire region occupied by the suspension, since the suspension is assumed to be statistically uniform) and $\boldsymbol{a}_{s}$ is the solid point acceleration relative to the mean value of the acceleration in $V$ (in the case studied, this value is uniform). The summations are over all the particles in the suspension.

As Batchelor states, for rigid particles $\boldsymbol{\Sigma}_{2}^{(p)}$ is zero; hence, since we are interested in rigid particles, we disregard this term. Also, he employs the weighting function $\psi(|x-z|)=1 / V$. Therefore, we can write:

$$
\begin{gathered}
\boldsymbol{\Sigma}_{1}^{(p)}(\boldsymbol{x}, t)=a \sum_{r}\left[\int_{\partial \Lambda_{r}} \boldsymbol{k}_{r}(\boldsymbol{z}, t) \boldsymbol{k}_{r}(\boldsymbol{z}, t) \cdot \boldsymbol{\sigma}_{e}(\boldsymbol{z}, t) d \boldsymbol{s}_{z}\right] \psi\left(\left|\boldsymbol{x}-\boldsymbol{z}_{r}\right|\right), \\
\boldsymbol{\Sigma}_{3}^{(p)}(\boldsymbol{x}, t)=\sum_{r}\left[\int_{\Lambda_{r}}\left(\boldsymbol{z}-\boldsymbol{z}_{r}\right) \rho_{s} \dot{\boldsymbol{u}}_{s} d \boldsymbol{z}\right] \psi\left(\left|\boldsymbol{x}-\boldsymbol{z}_{r}\right|\right) . \\
\boldsymbol{\Sigma}_{4}^{(p)}(\boldsymbol{x}, t)=\int_{\Lambda_{e}} \rho_{e} \boldsymbol{u}(\boldsymbol{z}, t) \boldsymbol{u}(\boldsymbol{z}, t) \psi(|\boldsymbol{x}-\boldsymbol{z}|) d \boldsymbol{z}+\sum_{r} \int_{\Lambda_{r}} \rho_{s} \boldsymbol{u}(\boldsymbol{z}, t) \boldsymbol{u}(\boldsymbol{z}, t) \psi(|\boldsymbol{x}-\boldsymbol{z}|) d \boldsymbol{z} .
\end{gathered}
$$

In Eq. (B8), we have replaced $\boldsymbol{a}_{s}$ with the solid point acceleration $\dot{\boldsymbol{u}}_{s}$, since the two differ by a constant vector that contributes nothing to the integral in Eq. (B8). 
$\boldsymbol{\Sigma}_{1}^{(p)}=n\langle\boldsymbol{A}\rangle_{p}$, the leading part of the particle-presence stress tensor; the part $\partial_{\boldsymbol{x}} \cdot\left(n\langle\boldsymbol{B}\rangle_{p}\right)$ is not recovered because is zero for statistically uniform suspensions. Moreover, as shown in Appendix A [see Eqs. (A21) and (A27)], $\boldsymbol{\Sigma}_{3}^{(p)}=-n\langle\boldsymbol{\Omega}\rangle_{p}$, a term that (as discussed) often can be neglected. Finally, it is as follows:

$$
\boldsymbol{\Sigma}_{4}^{(p)}=\varepsilon_{e} \rho_{e}\langle\hat{\boldsymbol{u}} \hat{\boldsymbol{u}}\rangle_{e}+\varepsilon_{s} \rho_{s}\langle\hat{\boldsymbol{u}} \hat{\boldsymbol{u}}\rangle_{s} .
$$

The collisional stress tensor does not appear in the particle stress tensor because Batchelor did not account for particle collisions. With these results, we then obtain:

$$
\boldsymbol{\Sigma}^{(p)}=n\langle\boldsymbol{A}\rangle_{p}+\varepsilon_{e} \rho_{e}\langle\hat{\boldsymbol{u}} \hat{\boldsymbol{u}}\rangle_{e}+\varepsilon_{s} \rho_{s}\langle\hat{\boldsymbol{u}} \hat{\boldsymbol{u}}\rangle_{s}-n\langle\boldsymbol{\Omega}\rangle_{p}
$$

We conclude that if $\left|\langle\boldsymbol{\omega}\rangle_{p}\right| /\left|\langle\boldsymbol{u}\rangle_{e}\right|$ is $O(1 / L)$, so that $n\langle\boldsymbol{\Omega}\rangle_{p}$ is $O\left(a^{2} / L^{2}\right)$, and if we neglect the kinetic stress terms, the particle stress tensor coincides with (the leading part of) the particle-presence stress tensor. But in general these two tensors differ.

\section{APPENDIX C: ON THE STEADY WIDE-GAP COUETTE FLOW SOLUTION PRESENTED IN MORRIS AND BOULAY}

In Sec. III B, to be consistent with the rest of our work, we have not used the notation adopted by Morris and Boulay [15]. We briefly repeat the analysis of the steady wide-gap Couette flow solution employing the original notation and clarifying a few additional aspects. The starting point is Eq. (31), which we now write as follows:

$$
\begin{aligned}
& \frac{d\left(r^{2} \Sigma_{f, r \theta}\right)}{d r}=0 ; \\
& \frac{d\left(r^{2} \Sigma_{p, r \theta}\right)}{d r}=0 .
\end{aligned}
$$

Both equations must be satisfied, but Morris and Boulay consider only the second. This is reported at page 1222, at the beginning of Section A, where the authors state "The steady or developed flow which occurs after particle migration is complete satisfies $\boldsymbol{\nabla} \cdot \boldsymbol{\Sigma}_{p}=\mathbf{0}$. In Secs. III A-C, we shall only need to consider the particle contributions to the stress and the subscript $p$ will be dropped from the particle stress."

So, based on what the authors say, from page 1223 when they write $\boldsymbol{\nabla} \cdot \boldsymbol{\Sigma}=\mathbf{0}$, they mean $\boldsymbol{\nabla} \cdot \boldsymbol{\Sigma}_{p}=$ 0. Now their closures are:

$$
\begin{aligned}
& \boldsymbol{\Sigma}_{f}=2 \eta \boldsymbol{E} ; \\
& \boldsymbol{\Sigma}_{p}=2 \eta \eta_{p}(\phi) \boldsymbol{E}-\eta \dot{\gamma} \boldsymbol{Q}(\phi),
\end{aligned}
$$

where $\eta$ is the fluid viscosity, a constant. As we said, Morris and Boulay did not consider Eq. (C1a), but this equation must be satisfied. Using Eq. (C2a) in Eq. (C1a) yields $d\left(r^{2} \dot{\gamma}\right) / d r=0$. Considering this result, let us use Eq. (C1b). From it, we obtain:

$$
2 \eta \eta_{p}(\phi) \frac{d\left(r^{2} \dot{\gamma}\right)}{d r}+2 \eta r^{2} \dot{\gamma} \frac{d \eta_{p}(\phi)}{d r}=0 \Rightarrow \frac{d \eta_{p}(\phi)}{d r}=0 .
$$

This means that $\eta_{p}(\phi)$ does not change with $r$, which in turn means that $\phi$ is uniform. That is, no migration has taken place, a result that is incorrect. Note that this problem is related to the closure of $\boldsymbol{\Sigma}_{f}$, in which the viscosity is a constant.

In Sec. III B, when modeling the steady wide-gap Couette flow, Morris and Boulay did find that migration occurs and did obtain good results because (a) they did not solve Eqs. (C1a) and (b) in the (un-tagged) equation below Eq. (28) of their article, they used $\eta_{s}(\phi)=1+\eta_{p}(\phi)$ instead of $\eta_{p}(\phi)$. Morris and Boulay solved only the following equation:

$$
\frac{d}{d r}\left[2 \eta \eta_{s}(\phi) r^{2} \dot{\gamma}\right]=0 .
$$


This is the very equation one obtains when using the following closures, where $\boldsymbol{\Sigma}_{p}$ is set to zero and the particle-presence stress is ascribed to the fluid effective stress tensor:

$$
\begin{aligned}
& \boldsymbol{\Sigma}_{f}=-\langle p\rangle_{e} \boldsymbol{I}+2 \eta \eta_{s}(\phi) \boldsymbol{E}-\eta \dot{\gamma} \boldsymbol{Q}(\phi) ; \\
& \boldsymbol{\Sigma}_{p}=\mathbf{0},
\end{aligned}
$$

where, in Eq. (C5a), we have accounted for the pressure term. So the equation that Morris and Boulay solved is consistent with what we argue in our article.

[1] P. Coussot and C. Ancey, Rheophysical classification of concentrated suspensions and granular pastes, Phys. Rev. E 59, 4445 (1999).

[2] F. Gadala-Maria and A. Acrivos, Shear-induced structure in a concentrated suspension of solid spheres, J. Rheol. 24, 799 (1980).

[3] Yu. A. Buyevich, Particle distribution in suspension shear flow, Chem. Eng. Sci. 51, 635 (1996).

[4] G. Segré and A. Silberberg, Radial particle displacements in poiseuille flow of suspensions, Nature 189, 209 (1961).

[5] S. R. Subia, M. S. Ingber, L. A. Mondy, S. A. Altobelli, and A. L. Graham, Modelling of concentrated suspensions using a continuum constitutive equation, J. Fluid Mech. 373, 193 (1998).

[6] R. Rao, L. Mondy, A. Sun, and S. Altobelli, A numerical and experimental study of batch sedimentation and viscous resuspension, Int. J. Numer. Methods Fluids 39, 465 (2002).

[7] Ju Min Kim, Song Geun Lee, and Chongyoup Kim, Numerical simulations of particle migration in suspension flows: Frame-invariant formulation of curvature-induced migration, J. Non-Newtonian Fluid Mech. 150, 162 (2008).

[8] T. Dbouk, L. Lobry, and E. Lemaire, Normal stresses in concentrated non-brownian suspensions, J. Fluid Mech. 715, 239 (2013).

[9] M. M. Reddy and A. Singh, Flow of concentrated suspension through oblique bifurcating channels, AIChE J. 60, 2692 (2014).

[10] R. B. Rebouças, I. R. Siqueira, P. R. de Souza Mendes, and M. S. Carvalho, On the pressure-driven flow of suspensions: Particle migration in shear sensitive liquids, J. Non-Newt. Fluid Mech. 234, 178 (2016).

[11] I. R. Siqueira and M. S. Carvalho, Particle migration in planar die-swell flows, J. Fluid Mech. 825, 49 (2017).

[12] T. Dbouk, Heat transfer and shear-induced migration in dense non-brownian suspension flows: Modeling and simulation, J. Fluid Mech. 840, 432 (2018).

[13] R. Jackson, The Dynamics of Fluidized Particles, Cambridge Monographs on Mechanics (Cambridge University Press, Cambridge, UK, 2000).

[14] R. Jamshidi, P. Angeli, and L. Mazzei, On the closure problem of the effective stress in the eulerianeulerian and mixture modeling approaches for the simulation of liquid-particle suspensions, Phys. Fluids 31, 013302 (2019).

[15] J. F. Morris and F. Boulay, Curvilinear flows of noncolloidal suspensions: The role of normal stresses, J. Rheol. 43, 1213 (1999).

[16] C. Cercignani, The Boltzmann Equation and Its Applications, Applied Mathematical Sciences (Springer, New York, 2012).

[17] P. R. Nott and J. F. Brady, Pressure-driven flow of suspensions: Simulation and theory, J. Fluid Mech. 275, 157 (1994).

[18] D. Gidaspow, Multiphase Flow and Fluidization: Continuum and Kinetic Theory Descriptions (Elsevier Science, Amsterdam, 1994).

[19] Yu. A. Buyevich, Particulate stresses in dense disperse flow, Industr. Eng. Chem. Res. 38, 731 (1999).

[20] D. Leighton and A. Acrivos, Viscous resuspension, Chem. Eng. Sci. 41, 1377 (1986). 
[21] D. Leighton and A. Acrivos, The shear-induced migration of particles in concentrated suspensions, J. Fluid Mech. 181, 415 (1987).

[22] R. J. Phillips, R. C. Armstrong, R. A. Brown, A. L. Graham, and J. R. Abbott, A constitutive equation for concentrated suspensions that accounts for shear-induced particle migration, Phys. Fluids A 4, 30 (1992).

[23] B. K. Chapman, Shear-induced Migration Phenomena in Concentrated Suspensions (University of Notre Dame, Notre Dame, South Bend, Indiana, 1990).

[24] A. W. Chow, S. W. Sinton, J. H. Iwamiya, and T. S. Stephens, Shear-induced particle migration in couette and parallel-plate viscometers: NMR imaging and stress measurements, Phys. Fluids 6, 2561 (1994).

[25] G. P. Krishnan, S. Beimfohr, and D. T. Leighton, Shear-induced radial segregation in bidisperse suspensions, J. Fluid Mech. 321, 371 (1996).

[26] D. Merhi, E. Lemaire, G. Bossis, and F. Moukalled, Particle migration in a concentrated suspension flowing between rotating parallel plates: Investigation of diffusion flux coefficients, J. Rheol. 49, 1429 (2005).

[27] A. W. Chow, J. H. Iwamiya, S. W. Sinton, and D. Leighton, Particle migration of non-Brownian, concentrated suspensions in a truncated cone-and-plate, in Proceedings of the Society of Rheology Meeting (Society of Rheology, Sacramento, California, 1995).

[28] P. R. Nott, E. Guazzelli, and O. Pouliquen, The suspension balance model revisited, Phys. Fluids 23, 043304 (2011).

[29] Yu. A. Buyevich and S. K. Kapbsov, Segregation of a fine suspension in channel flow, J. Non-Newt. Fluid Mech. 86, 157 (1999).

[30] G. J. Hwang and H. H. Shen, Modeling the solid phase stress in a fluid-solid mixture, Int. J. Multiphase Flow 15, 257 (1989).

[31] H. M. Vollebregt, R. G. M. van der Sman, and R. M. Boom, Suspension flow modeling in particle migration and microfiltration, Soft Matter 6, 6052 (2010).

[32] F. Municchi, P. P. Nagrani, and I. C. Christov, A two-fluid model for numerical simulation of sheardominated suspension flows, Int. J. Multiphase Flow 120, 103079 (2019).

[33] M. Sarabian, Mohammadhossein Firouznia, B. Metzger, and S. Hormozi, Fully developed and transient concentration profiles of particulate suspensions sheared in a cylindrical couette cell, J. Fluid Mech. 862, 659 (2019).

[34] R. Jackson, Locally averaged equations of motion for a mixture of identical spherical particles and a newtonian fluid, Chem. Eng. Sci. 52, 2457 (1997).

[35] D. Z Zhang and A. Prosperetti, Momentum and energy equations for disperse two-phase flows and their closure for dilute suspensions, Int. J. Multiphase Flow 23, 425 (1997).

[36] G. K. Batchelor, The stress system in a suspension of force-free particles, J. Fluid Mech. 41, 545 (1970).

[37] P. Lettieri and L. Mazzei, Challenges and issues on the cfd modeling of fluidized beds: A review, J. Comput. Multiphase Flows 1, 83 (2009).

[38] A. Einstein, Eine neue bestimmung der moleküldimensionen, Ann. Phys. 324, 289 (1906).

[39] I. Drijer, T. van de Laar, H. M. Vollebregt, and C. G. P. H. Schroën, From highly specialised to generally available modeling of shear induced particle migration for flow segregation based separation technology, Separat. Purificat. Technol. 192, 99 (2018).

[40] M. Manninen, V. Taivassalo, and S. Kallio, On the Mixture Model for Multiphase Flow (VTT Publications, Technical Research Center of Finland, Espoo, 1996).

[41] L. Mazzei and P. Lettieri, A drag force closure for uniformly dispersed fluidized suspensions, Chem. Eng. Sci. 62, 6129 (2007).

[42] T. Elson L. Mazzei, P. Lettieri and D. Colman, A revised monodimensional particle bed model for fluidized beds, Chem. Eng. Sci. 61, 1958 (2006).

[43] C.-Y. Wen and Y. H. Yu, Mechanics of fluidization, Chem. Eng. Progr. Symp. Ser. 62, 100 (1966).

[44] D. Lhuillier, Migration of rigid particles in non-brownian viscous suspensions, Phys. Fluids 21, 023302 (2009). 
[45] D. Z. Zhang and A. Prosperetti, Averaged equations for inviscid disperse two-phase flow, J. Fluid Mech. 267, 185 (1994).

[46] J. G. Savins and A. B. Metzner, Radial (secondary) flows in rheogoniometric devices, Rheol. Acta 9, 365 (1970).

[47] J. J. J. Gillissen and H. J. Wilson, Modeling sphere suspension microstructure and stress, Phys. Rev. E 98, 033119 (2018).

[48] L. Mazzei, Eulerian Modeling and Computational Fluid Dynamics Simulation of Mono and Polydisperse Fluidized Suspensions, Ph.D. thesis, UCL (University College London, London, 2008).

[49] L. Mazzei, Recent advances in modeling gas-particle flows, in Handbook of Multiphase Flow Science and Technology, edited by Guan Heng Yeoh (Springer Singapore, Singapore, 2017) pp. 1-43.

[50] Andreas ten Cate Sankaran Sundaresan, Analysis of the flow in inhomogeneous particle beds using the spatially averaged two-fluid equations, Int. J. Multiphase Flow 32, 106 (2006).

[51] H. R. Norouzi, R. Zarghami, R. Sotudeh-Gharebagh, and N. Mostoufi, Coupled CFD-DEM Modeling: Formulation, Implementation and Application to Multiphase Flows (Wiley, New York, 2016).

[52] L. Mazzei, Recent advances in modeling gas-particle flows, in Handbook of Multiphase Flow Science and Technology, edited by Guan Heng Yeoh (Springer, Berlin, 2017), pp. 1-43. 